Accepted to the Astronomical Journal

\title{
The Membership and Distance of the Open Cluster Collinder 419
}

\author{
Lewis C. Roberts, Jr. ${ }^{1}$, Douglas R. Gies ${ }^{2}$, J. Robert Parks ${ }^{2}$, Erika D. Grundstrom ${ }^{3,8}$, M. \\ Virginia McSwain ${ }^{4,8}$, David H. Berger ${ }^{5}$, Brian D. Mason ${ }^{6}$, Theo A. ten Brummelaar ${ }^{7}$, and \\ Nils H. Turner ${ }^{7}$
}

\begin{abstract}
The young open cluster Collinder 419 surrounds the massive O star, HD 193322, that is itself a remarkable multiple star system containing at least four components. Here we present a discussion of the cluster distance based upon new spectral classifications of the brighter members, $U B V$ photometry, and an analysis of astrometric and photometric data from the UCAC3 and 2MASS catalogs. We determine an average cluster reddening of $E(B-V)=0.37 \pm 0.05$ mag and a cluster distance of $741 \pm 36 \mathrm{pc}$. The cluster probably contains some very young stars that may include a reddened M3 III star, IRAS $20161+4035$.
\end{abstract}

Subject headings: stars: individual (HD 193322, IRAS 20161+4035) — stars: early-type - open clusters and associations: individual (Collinder 419)

\footnotetext{
${ }^{1}$ Jet Propulsion Laboratory, California Institute of Technology, 4800 Oak Grove Drive, Pasadena CA 91109; lewis.c.roberts@jpl.nasa.gov

${ }^{2}$ Center for High Angular Resolution Astronomy, Department of Physics and Astronomy, Georgia State University, P.O. Box 4106, Atlanta, GA 30302-4106; gies@chara.gsu.edu, parksj@chara.gsu.edu

${ }^{3}$ Physics and Astronomy Department, Vanderbilt University, 6301 Stevenson Center, Nashville, TN 37235; erika.grundstrom@vanderbilt.edu

${ }^{4}$ Department of Physics, Lehigh University, 16 Memorial Drive East, Bethlehem, PA 18015; mcswain@lehigh.edu

${ }^{5}$ System Planning Corporation, 3601 Wilson Blvd, Arlington, VA 22201; dberger@sysplan.com

${ }^{6}$ US Naval Observatory, 3450 Massachusetts Avenue, NW, Washington, DC 20392-5420; bdm@usno.navy.mil

${ }^{7}$ Center for High Angular Resolution Astronomy, Georgia State University, Mt. Wilson, CA 91023; theo@chara-array.org, nils@chara-array.org

${ }^{8}$ Visiting Astronomer, Kitt Peak National Observatory, National Optical Astronomy Observatory, operated by the Association of Universities for Research in Astronomy, Inc., under contract with the National Science Foundation.
} 


\section{Introduction}

Collinder 419 is an open cluster in the constellation Cygnus. It was first designated as Barnard 794 (Barnard 1927), but the accepted name comes from the work of Collinder (1931) who classified the cluster as a $\mu$ Normae cluster. This is an intermediate type of grouping between multiple star systems and open clusters. The prototype cluster NGC 6169 contains the bright star $\mu$ Normae $=$ HD 149038 and a host of surrounding fainter stars. In the case of Collinder 419, the bright central star is HD $193322=$ HR $7767=$ HIP $100069=$ WDS 20181+4044. HD 193322 is a multiple star system with a close visual companion STF $2666 \mathrm{AB}$ (separation of 2".9 and probably orbitally bound) and two more distant companions, STF $2666 \mathrm{AC}\left(34^{\prime \prime} .0\right)$ and TAR $5 \mathrm{AD}$ (49!'6), which, if bound have very long orbital periods. McAlister et al. (1987) discovered that the A component is a speckle binary, CHR 96 Aa,Ab. An approximately 31 year orbital period for this pair was computed by Hartkopf et al. (1993). In addition, one of the components of the speckle binary is a spectroscopic binary with a $311.03 \pm 0.25$ day period (Fullerton 1990; McKibben et al. 1998). Fullerton (1990) also obtained a spectrum of the B component that suggests that it may also be a spectroscopic binary. Thus, the central AB pair may contain as many as five individual stars.

The primary of the system, Aa1, has a spectral type of O9 V:((n)) (Walborn 1972), with the suffix indicating slightly broadened lines. McKibben et al. (1998) tentatively identify the close spectroscopic companion Aa2 and the B component all as B-type stars. The magnitude difference of the speckle pair Aa,Ab is $\triangle V=1.2 \pm 0.5$ (Mason et al. 2009) which suggests that $\mathrm{Ab}$ is also an early B-type star. Both the Aa2 and Ab companions offer us the means to determine mass for the O star component Aa1 through measurement of the orbital motion. By themselves, neither an orbit of the Aa,Ab pair nor the spectroscopic Aa1,Aa2 pair will yield a mass, but, in conjunction with a distance determination, a mass can be determined. The Hipparcos Catalogue (Perryman et al. 1997) has become the standard source for parallaxes, but it may be unreliable for some double stars (Shatskii \& Tokovinin 1998) and also for distant O stars (Schröder et al. 2004). Until a new space-based parallax

engine is available sometime in the next decade we are left with classical techniques as the best way to determine O-star distances.

We are currently pursuing efforts to determine the orbits of the central triple through speckle and optical long baseline interferometry (Turner et al. 2008). Here we present a new assessment of the distance to the cluster that we will use in the forthcoming orbit and mass determination. In $\S 2$ we present spectral classifications for the brighter members, and we show fits of their spectral energy distributions that provide estimates of reddening and angular size. We then describe in $\S 3$ an astrometric and photometric study of cluster membership based upon data in the UCAC3 catalog (Zacharias et al. 2010) and the 2MASS 
catalog (Cutri et al. 2003). We also present new $U B V$ photometry of the fainter cluster members in $\S 4$. Our results are summarized in $\S 5$.

\section{Spectroscopy and Reddening of the Brighter Stars}

We obtained spectra for five of the brighter stars in the cluster in order to determine spectral classifications and to estimate the intrinsic colors of the stars. One of us (MVM) obtained spectra of the four blue stars of the central multiple system using the Kitt Peak National Observatory (KPNO) $2.1 \mathrm{~m}$ telescope and Cassegrain focus, GoldCam CCD Spectrograph. These spectra were made during 2005 October and November with the No. 47 grating (831 grooves $\mathrm{mm}^{-1}$ ) in second order to obtain a resolving power $\lambda / \delta \lambda \sim 3000$. The observed wavelength range was 4050 - $4950 \AA$, a region that includes numerous $\mathrm{H}$ Balmer, He I, and He II lines in O- and B-type spectra. The data were reduced using standard routines in IRAF9. The flux rectified spectra were compared directly to spectra from the atlas of Valdes et al. (2004) in order to estimate the spectral classification based upon the strengths of the H Balmer, Ca II, He I, and Mg II lines. Our results are given in Table 1 for the B, C, and D components of HD 193322. See Figures 1 - 3 for the comparision between the target stars and the template spectra. Our spectra for the combined flux of Aa1, Aa2, and Ab agree with the O9 V:((n)) classification from Walborn (1972), which is listed in Table 1. These classifications all agree within a subtype with earlier results (Burnichon 1975; Hoffleit 1982; Abt \& Cardona 1983).

The very red star IRAS $20161+4035$ is the brightest object in the $K_{s}$-band in this vicinity, and we were curious to determine if indeed it is related to the cluster. One of us (EDG) obtained three spectra of the object with the KPNO 0.9 m Coude Feed Telescope in 2004 October. These spectra cover the range from 6460 to $7140 \AA$ with a resolving power $\lambda / \delta \lambda \sim 9700$. The average spectrum is compared to several other cool star spectra from Valdes et al. (2004) (also made with the KPNO Coude Feed telescope) in Figure 4, We made a digital comparison with all the M2 - M5 spectra in the Valdes et al. spectral atlas, and the best fit (based mainly on the appearance of the strong $\mathrm{TiO}$ bands in this spectral region) was the spectrum of the M3 II star HD 40239 (although there were a number of other M3 III spectra which made an almost equally good fit). Figure 4 shows the good overall match made with the M2 II and M3 III giants compared to the dwarf and supergiant spectra.

\footnotetext{
${ }^{9}$ IRAF is distributed by the National Optical Astronomical Observatory, which is operated by the Association of Universities for Research in Astronomy, Inc. (AURA), under cooperative agreement with the National Science Foundation.
} 
We also measured the $\mathrm{H} \alpha$ equivalent width in these and similar stars to help establish the luminosity class (Eaton 1995). We found that among stars of similar spectral type that the mean equivalent width of $\mathrm{H} \alpha$ ranged from $0.76 \pm 0.04 \AA$ for 2 main sequence stars, to $1.59 \pm 0.08 \AA$ for 4 giant stars, and up to $1.89 \pm 0.04 \AA$ for 3 supergiant stars. The measured $\mathrm{H} \alpha$ equivalent width of IRAS $20161+4035,1.38 \AA$, clearly places the star among those in the giant luminosity class, and we adopted this assignment in Table 1 . Note that the spectrum of IRAS $20161+4035$ shows the strongest Li I $\lambda 6707$ feature among the spectra illustrated in Figure 4 .

We can use these spectral classifications to estimate the intrinsic colors of the targets, compare these with observed multi-color observations, and find the reddening $E(B-V)$ in each case. However, the available near-IR 2MASS photometry for HD 193322 corresponds to the total flux for the entire Aa1, Aa2, Ab, and B complex. Thus, we need to make some assumptions about the relative flux contributions of each component in each filter band to derive magnitudes and fluxes for the individual stars. The magnitude differences of the AB pair were determined in the Johnson $B V R I$ bands through adaptive optics imaging by ten Brummelaar et al. (2000). Unfortunately, much less is known about the A sub-components. Mason et al. (2009) found a magnitude difference of $\triangle V=1.2 \pm 0.5$ mag for the Aa, $\mathrm{Ab}$ speckle pair, and McKibben et al. (1998) used a statistical method to estimate the mass ratio and magnitude difference $\triangle V=1.3 \mathrm{mag}$ for the Aa1,Aa2 pair in the spectroscopic binary. These magnitude differences (plus $\triangle V$ for the $\mathrm{AB}$ pair from ten Brummelaar et al. 2000) lead to $V$-band flux ratios relative to star Aa1 that are given in Table 2. To derive the flux ratios in other bands, we estimated their colors according to their spectral classification and the color calibration from Wegner (1994). We assumed that the Aa2 and Ab stars are main sequence objects and then estimated their spectral types (B1.5 V and B1 V, respectively) based on their magnitude differences relative to Aa1 (using the absolute magnitude versus spectral type relation for main sequence stars from Lesh 1979). Table 2 lists the adopted flux ratio estimates for the sub-components (which agree with those from ten Brummelaar et al. for the Johnson $R, I$ bands, but not for their $B$-band result for which component $\mathrm{B}$ is unusually faint in $B$ relative to the other bands). We used these flux ratios to determine the magnitudes of Aa1 and B, the stars with reliable spectral classifications, in the following analysis. Note that the current flux ratio uncertainties probably introduce an error of \pm 0.2 mag into the error budget for the magnitudes of these two stars.

We estimated the reddening and angular sizes of the five bright stars listed in Table 1 by comparing their observed fluxes with reddened model spectral energy distributions. The optical magnitudes were taken from the work of ten Brummelaar et al. (2000; for components Aa1 and B), Burnichon \& Garnier (1976; for components C and D) and Droege et al. (2006; plus $U B V$ magnitudes given in $\S 4$ for IRAS 20161+4035), and these were combined with 
2MASS magnitudes for the near-IR (Cutri et al. 2003). The magnitudes were transformed to fluxes using calibrations from Colina et al. (1996; Johnson $U, B, V$ ), Bessell et al. (1998; Cousins $I_{c}$ ), and Cohen et al. (2003; 2MASS $\left.J, H, K_{s}\right)$. We used Kurucz model atmospheres 10 (assuming $\log g=4.0$, solar abundances, and a microturbulent velocity of $2 \mathrm{~km} \mathrm{~s}^{-1}$ ) for temperatures from the spectral calibration of Böhm-Vitense (1981; see Table 1) to create low resolution versions of the unreddened spectral energy distributions (SEDs). However, for the case of IRAS 20161+4035 (M3 III), we adopted a flux distribution from the MARCS code 11 (Gustafsson et al. 2008) for a model with $T_{\text {eff }}=3500 \mathrm{~K}, \log g=1.0$, solar abundances, and a microturbulent velocity of $5 \mathrm{~km} \mathrm{~s}^{-1}$. We then fit the observed fluxes with model SEDs reddened according to the formulation from Fitzpatrick (1999). Because the stars have low reddening and limited short-wavelength coverage, it is difficult to obtain an independent estimate of the ratio of total-to-selective extinction $R$, so we adopted the default value $R=3.1$ throughout. The derived reddening $E(B-V)$ and limb-darkened angular diameter $\theta_{L D}$ are given in Table 1 . The blue stars yield a consistent reddening of $E(B-V)=0.29 \pm 0.01$ mag, which is significantly less than that found for IRAS $20161+4035, E(B-V)=0.74 \pm 0.04$ mag. The SED for this red star is shown in Figure 5, and we see that there appears to be an IR flux-excess of $48 \pm 10 \%$ near $12 \mu \mathrm{m}$, based upon data from the MSX (Egan \& Price 1996) and IRAS12 missions. We argue below ( $\$ 5$ ) that the higher reddening and IR-excess may be related to the youth of this star.

\section{Astrometric and Photometric Data from UCAC3 and 2MASS}

With the recent release of the third U. S. Naval Observatory CCD Astrograph Catalog 13 (UCAC3; Zacharias et al. 2010), we now have access to a large collection of astrometric and photometric data that can be used to search for additional cluster members. We began by selecting all the stars in UCAC3 with complete sets of proper motion and photometric data that are found within a 0.25 radius of HD 193322, the nominal center of $\mathrm{Cr} 419$. We show in Figure 6 the average surface density of star counts within annuli centered on the position of HD 193322. The larger surface density associated with the cluster extends outwards to at least 0.10 , and we set the outer boundary on cluster membership at 0.16 to avoid including too many field stars at large distance. Figure 7 shows a 0.25 field centered on HD 193322

\footnotetext{
${ }^{10}$ http://kurucz.harvard.edu/

${ }^{11}$ http://marcs.astro.uu.se/

${ }^{12}$ Infrared Astronomical Satellite Catalogs, 1988; The Point Source Catalog, version 2.0, NASA RP-1190

${ }^{13}$ http://www.usno.navy.mil/USNO/astrometry/optical-IR-prod/ucac
} 
and shows the low stellar density of the cluster. The image is from the near-IR plates of Second Palomar Observatory Sky Survey (POSS2-IR) (Reid et al. 1991). Figure 8 shows the distribution of proper motions of stars found within this radius. The circle in the diagram

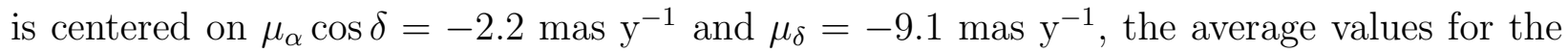
bright cluster stars (HD 193322A, C, D, and HD 228810). There appears to be a bimodal distribution in proper motion in this direction, so we selected only those stars within the circle, i.e., those with a combined proper motion difference from the cluster mean of less than 11 mas $^{-1}$ (the RMS of the UCAC3 proper motion errors and the expected range within the cluster itself). The constraints of position and proper motion reduced the potential sample to a group of 96 stars.

The histogram in Figure 6 also demonstrates that there will be a significant number of field stars in this sample. To estimate this number, we applied the same proper motion constraint to the UCAC3 sample within a separation range of 0.16 to 0.24 , and this indicates that the surface density of field stars in this direction with similar proper motions to the bright cluster stars is $1000 \pm 100$ stars per $\mathrm{deg}^{2}$. Thus, we might expect to find some $83 \pm 8$ field stars in the 0.16 radius circle centered on HD 193322. This exercise suggests that the actual number of cluster stars is small (perhaps 96 total - 83 field $=13$ cluster stars) and that the sample will be dominated by other field stars.

We next considered the photometric data in UCAC3, which consists of 2MASS photometry and the UCAC3 red-band photometry that we will refer to as $R_{u}$ (to distinguish it from the Johnson and Cousins counterparts). This filter has a bandpass from 579 to $642 \mathrm{~nm}$ that falls between the Johnson $V$ and Cousins $R_{c}$ bands. The UCAC3 catalog reports magnitudes from centroid fit models and aperture photometry, and we used the latter in our analysis. The UCAC3 listing for HD 193322 corresponds to the total flux from components Aa1, Aa2, $\mathrm{Ab}$, and $\mathrm{B}$, and we used the flux ratios given in Table 2 to determine the corresponding magnitudes for stars Aa1 and B only. Our goal is to compare the magnitudes of the candidate member stars with model predictions for given assumptions about the reddening $E(B-V)$ and distance modulus DM, in order to find values of these parameters that lead to the best fit of the photometry.

We adopted model isochrones for the cluster from the evolutionary models of Bertelli et al. (1994) and Marigo et al. (2008)14. We selected an isochrone for solar metallicity and a cluster age of $7 \mathrm{Myr}$, the value proposed by Kharchenko et al. (2005) and one that is consistent for a time of flight from Cr 419 of the runaway star 68 Cyg (Schilbach \& Röser 2008). The isochrones were produced for a number of photometric bands including Johnson

\footnotetext{
${ }^{14} \mathrm{http} / / /$ stev.oapd.inaf.it/cmd
} 
$V$, Cousins $R_{c}$, and 2MASS $J, H, K_{s}$.

We need to transform the model optical magnitudes into the $R_{u}$ system in order to compare them with the UCAC3 values. We used a sample of 402 photometric standard stars from Landolt (1992) that appear in UCAC3 to arrive at a transformation equation from Johnson $V$ and Cousins $R_{c}$ to $\mathrm{UCAC} 3 R_{u}$,

$$
R_{u}=R_{c}+0.292+0.372\left(V-R_{c}\right)+0.054\left(R_{c}-13\right)
$$

which successfully fits the observed UCAC3 $R_{u}$ magnitudes over the range $R_{u}=8-17$ with a standard deviation of 0.116 mag. The color term in the equation shows how the $R_{u}$ magnitude changes with stellar color because of the difference in the effective wavelengths of the $R_{u}$ and $R_{c}$ bands. The final term on the right hand side reflects a small systematic difference between the Landolt and UCAC3 systems that is approximately equal to the scatter in the relation at the bright and faint ends of the sample.

We can apply a few other constraints to limit the number of field stars that may exist within the astrometric group of cluster candidates. Figure 9 shows the near-IR color-color diagram for the sample together with unreddened (solid line) and reddened (dashed line) versions of the cluster isochrone. The numbers beside the unreddened version indicate the stellar mass corresponding to the color pair, and massive stars have colors in the lower part of the diagram. The reddening vector direction is indicated by the dotted line. Comerón et al. (2002) and others have argued that the reddening-free parameter $Q=(J-H)-1.94 *\left(H-K_{s}\right)$ (Fitzpatrick 1999; Indebetouw et al. 2005) provides an excellent means to identify massive, reddened stars that typically have $Q \approx 0.0$. We decided to apply this criterion by restricting the sample to those massive stars with $Q<0.116$, the model value for a star of mass $M=1.4 M_{\odot}$. Imposing this limit will mean that we lose fainter, red cluster members, but, on the other hand, we will also remove cool background giants and cooler, low mass, foreground stars that do not belong to the cluster (for example, those stars found near the unreddened isochrone between 0.8 and $1.4 M_{\odot}$ ).

We also applied a faint limit constraint, $R_{u}<15$, to avoid stars with large magnitude errors. Since our initial sample was drawn from UCAC3 stars with complete photometric coverage, the result is that this sample tends towards stars with equal magnitude in all filters at the faint end. This group probably includes foreground A-type stars, so applying the faint limit will help us remove such stars from consideration.

We show two versions of the color - magnitude diagram in Figures 10 \& 11. Both of these show evidence of a main sequence group at the blue end, but both also show a large population of redder stars that lie above the expected main sequence. In order to remove such targets, we restricted the sample to those with $R_{u}-J<1.35$. This constraint, 
together with the $Q$ limit described above, will result in a sample without any low mass, main sequence members.

Our final constraint is based on how closely the magnitudes match the model isochrone values. For any given choice of $E(B-V)$ and $\mathrm{DM}$, we formed an average $\chi_{\nu}^{2}$ statistic between all the observed and model magnitudes (reddened according to the relations given by Fitzpatrick 1999) for each mass point along the model isochrone. The best fit (lowest $\chi_{\nu}^{2}$ ) model point was taken as an appropriate mass for the specific star. Those stars with magnitudes and colors very different from the main sequence values will have a large minimum $\chi_{\nu}^{2}$, and we restricted our final sample of main sequence cluster members to those with $\chi_{\nu}^{2}<35$, a value high enough to include those cases where the observational photometric errors are underestimated. The final sample consists of 16 stars that are listed in Table 3 and are indicated in Figures 9 - 11 by plus signs. Note that we make the tacit assumption that the same reddening can be applied to all cluster members, and this can only be true in some average sense. There may be a reddening gradient across this field (Schlegel et al. 1998) and some of the youngest objects may suffer from circumstellar dust reddening. Nevertheless, until spectroscopy is available for many stars and their intrinsic colors found, we must accept this working hypothesis.

We performed a grid search over a range of $E(B-V)$ and DM to find those values that minimized the average $\chi_{\nu}^{2}$ value for the selected cluster stars. The best fit was made with $E(B-V)=0.37 \pm 0.05 \mathrm{mag}$ and $\mathrm{DM}=9.35 \pm 0.03 \mathrm{mag}$, yielding an average photometric statistic of $\chi_{\nu}^{2}=4.4$. The isochrones for these parameters are plotted in Figures 9-11, and the corresponding main sequence masses from the isochrone are listed in the final column of Table 3. Our derived reddening estimate is mainly consistent with earlier estimates for the cluster and star $(E(B-V)=0.34$, Kharchenko et al. 2005; $E(B-V)=0.345$, Burnichon 1975; $E(B-V)=0.38$, Schröder et al. 2004; $E(B-V)=0.41$, Cardelli et al. 1989). The reddening is slightly larger than that found by SED fitting for the blue stars (Table 1), but we suspect that the differences arise in the variable reddening across the cluster. We also repeated the analysis using an isochrone for a younger cluster (age $3 \mathrm{Myr}$ ), but the results were unchanged because the predicted colors and magnitudes were only significantly different for the most massive star HD 193322Aa1 (the predicted colors are marginally closer to the observed ones for the $7 \mathrm{Myr}$ isochrone).

There are many stars that are plotted above the main sequence in Figures [10 \& 11. Some of these stars may belong to a massive and more distant population. For example, in the infrared color-color diagram (Fig. 9), there appears to be a reddened group of OB stars near $H-K_{s}=0.2$ and $J-H=0.4$. An independent analysis of the 2MASS magnitudes of stars in the vicinity of $\mathrm{Cr} 419$ by David Turner (priv. communication) suggests that these 
correspond to objects with $E(B-V)=1.5$ at a distance of $1.6 \mathrm{kpc}$ that may be associated with the nearby cluster Berkeley 87 (Turner \& Forbes 1982; Massey et al. 2001). We show how the $7 \mathrm{Myr}$ isochrone would appear for such reddened and distant stars as a dotted line in Figures 10 and 11, and many of the stars appearing above the $\mathrm{Cr} 419$ main sequence are close to the predicted colors and magnitudes of a distant population. Cr 419 is probably young enough that it may host pre-main sequence stars that would also appear above the main sequence. Dashed lines in Figure 10 and 11 indicate the pre-main sequence isochrone

for an age of $7 \mathrm{Myr}$ from the work of Siess et al. (2000) transformed to the distance and reddening of $\mathrm{Cr} 419$, and indeed we find many examples of stars close to the predicted track. We suspect that redder stars appearing in these color-magnitude diagrams probably include some cluster pre-main sequence stars and large numbers of distant massive stars and other line-of-sight field stars.

\section{4. $U B V$ Photometry}

We also obtained new $U B V$ photometry of the central region of the cluster to explore the optical colors of the fainter stars. One of us (JRP) collected CCD images of Collinder 419 in 2006 October with the Emory University Observatory, DFM 0.6 m Cassegrain telescope15. The detector was an Apogee Ap47 $1024 \times 1024$ pixel CCD camera. In order to concentrate the signal for fainter sources, the camera was set to a $2 \times 2$ binning mode with a net pixel scale of $1^{\prime \prime} .1$ pixel $^{-1}$ and a 9'.5 $\times 9$ 9.5 field of view. The images of the brightest stars were saturated in individual frames in order to obtain a stronger signal on the faintest sources. Thirty frames were collected through each Johnson $U B V$ filter. In addition, standard stars from charts 140, 141, and 148 of Landolt (1992) were observed over a large range in air mass to transform the instrumental magnitudes to the standard system.

All the frames were debiased, dark subtracted, and flat fielded using standard routines in IRAF. The images were combined using IMCENTROID and IMCOMBINE to build a master image in each filter. Photometric measurements of the standard stars were made with aperture photometry routines in IRAF. The transformation coefficients were computed using the IDL procedure transf1.pro, written by Marc W. Buie. This procedure uses the general formula

$$
m_{O}=m_{i}-k^{\prime} X-k^{\prime \prime} C X+e C+Z
$$

where $m_{0}$ is the standard magnitude, $m_{i}$ is the instrumental magnitude, $k^{\prime}$ is the first order extinction coefficient, $X$ is the airmass, $k^{\prime \prime}$ is the second order extinction coefficient, $C$ is a

\footnotetext{
${ }^{15}$ http://www.physics.emory.edu/astronomy/observatory.html
} 
color index, $e$ is a color coefficient, and $Z$ is the magnitude zero point. The instrumental magnitudes of the stars in the Collinder 419 frames were measured using PSF fitting routines in IRAF, and the relation between the PSF and aperture measurements was determined for ten well isolated stars in the field. The transformation equations were then used to determine Johnson magnitudes for the cluster stars. The errors in the transformation coefficients were added in quadrature with the instrumental magnitude errors to obtain the net observational errors (approximately 0.04, 0.03, and $0.02 \mathrm{mag}$ in $U B V$, respectively). The results are listed in Table 4.

We show in Figure 12 the color - magnitude diagram $(B-V, V)$ for 43 of the 79 stars measured that meet the proper motion limits for cluster membership described above (§3). Most of the proposed cluster members ( $\$ 3$; Table 3) were too bright and/or outside of the field of view for our $U B V$ photometric measurements, and we have $U B V$ photometry for only 5 of the 16 proposed members (indicated by plus signs in Fig. 8 and by "Yes" in the final column of Table 4). Figure 12 also shows the main sequence relation from the models of Bertelli et al. (1994) and Marigo et al. (2008) for our derived estimates of $E(B-V)=0.37$ mag and DM $=9.35 \mathrm{mag}$, and this relation appears to be consistent with our optical photometry. There are a number of stars that have magnitudes indicative of lower mass, main sequence stars, but there are many objects with a position above the main sequence with a distribution similar to that seen in the red and near-infrared color - magnitude diagrams (Figs. 10 and 11). Figure 13 shows the optical color - color diagram $(B-V, U-B)$ for the subset with complete $U B V$ measurements and within the proper motion limits for membership. We also show both unreddened and reddened versions of the model colors. Again, there is evidence for some lower main sequence members (towards the middle and lower sections of the dashed line), but there is also a group of stars with colors near $(B-V, U-B) \approx(1.0,0.0)$ that are characteristic of B-stars with a reddening $E(B-V) \approx 1.0$ mag. Most of these stars also have positions in the near-IR color - color diagram associated with reddened, intermediate mass stars (Fig. 9; $\left(H-K_{s}, J-H\right) \approx(0.15,0.30)$ ).

\section{Discussion}

The distance we obtained from the photometric analysis of $d=741 \pm 11 \mathrm{pc}$ (formal error) does not account for possible systematic errors in the model isochrone magnitudes. By comparing the $\left(R_{c}-J, J\right)$ color-magnitude diagrams for a 7 Myr cluster from Marigo et al. (2008) with that from Lejeune \& Schaerer (2001), we estimate that the model uncertainties amount to $\triangle J \approx 0.10 \mathrm{mag}$ over the main sequence range of interest, so we add this error in quadrature with the fitting errors to obtain $d=741 \pm 36$ pc. We can check this with distances 
derived from the angular sizes given in Table 1. This distance estimate depends on the ratio of the physical diameter to the angular diameter, and for this comparison, we estimated the stellar radii for HD 193322B, C, and D from the model isochrone values for main sequence stars with our adopted effective temperatures (Table 2). We could not use this relation for HD 193322Aa1 because the model isochrone did not extend to stars quite this hot, so we instead adopted a radius of $R=7.53 R_{\odot}$ from the spectral calibration for main sequence stars from Martins et al. (2005). We estimated a radius of $R=75 R_{\odot}$ for the M3 III star IRAS 20161+4035 from the calibration for giants by van Belle et al. (1999). The resulting distances are listed in the final column of Table 1 , and the average, $d=766 \pm 51 \mathrm{pc}$, agrees with the photometric result. Our results confirm the only previous distance estimate for the cluster from Kharchenko et al. (2005) of 740 pc (no error quoted). The cluster distance also agrees within errors with the Hipparcos distance for HD 193322 of $600_{-110}^{+150}$ pc (van Leeuwen 2007).

We suspect that some of the red stars that meet the spatial and proper motion constraints of cluster membership but lie above the main sequence are very young objects. A similar population of bright, red objects has been reported for other young star clusters containing O stars (Kumar et al. 2004; Comerón \& Pasquali 2005; Carlson et al. 2007; Negueuela et al. 2007; Comerón et al. 2008). These stars may include IR-excess objects with remnant disks like the Herbig Ae/Be stars. Although it is possible that the red object, IRAS $20161+4035$, is an old star that is coincidentally passing through the vicinity of $\mathrm{Cr} 419$, we suspect that it may also be a very young member of the cluster, despite its evolved appearance. The star's large reddening and IR-excess both suggest that it is immersed in natal dust, and its strong Li I $\lambda 6707$ and high luminosity may be indicative of youth. Kumar et al. (2004) identified three other cases of luminous, late-type giants that are found in young clusters. These cool giants may represent another kind of spectroscopic manifestation of young stellar objects.

We thank Todd Henry, Adric Riedel, and Russel White for helpful comments about M-star spectra, and we also thank Bill Binkert of the KPNO staff for his assistance at the KPNO Coude Feed Telescope. We are grateful to David Turner for discussions about field contamination by distant massive stars. This publication made use of data products from the Two Micron All Sky Survey, which is a joint project of the University of Massachusetts and the Infrared Processing and Analysis Center/California Institute of Technology, funded by the National Aeronautics and Space Administration and the National Science Foundation. It also used images from the Second Palomar Observatory Sky Survey (POSS-II), which was made by the California Institute of Technology with funds from the National Science Foundation, the National Geographic Society, the Sloan Foundation, the Samuel Oschin 
Foundation, and the Eastman Kodak Corporation. We also made use of the Washington Double Star Catalog, maintained at the U. S. Naval Observatory, and the SIMBAD database, operated by the CDS in Strasbourg, France. This material is based upon work supported by the National Science Foundation under Grant No. AST-0606861. Institutional support has been provided from the GSU College of Arts and Sciences and from the Research Program Enhancement fund of the Board of Regents of the University System of Georgia, administered through the GSU Office of the Vice President for Research. A portion of the research was carried out at the Jet Propulsion Laboratory, California Institute of Technology, under a contract with the National Aeronautics and Space Administration. 


\section{REFERENCES}

Abt, H. A., \& Cardona, O. 1983, ApJ, 272, 182

Barnard, E. E. 1927, A photographic atlas of selected regions of the Milky Way, ed. E. B. Frost \& M. R. Calvert (Carnegie Inst. Washington Publ. 247) (Washington DC: Carnegie Inst.)

Bertelli, G., Bressan, A., Chiosi, C., Fagotto, F., \& Nasi, E. 1994, A\&AS, 106, 275

Bessell, M. S., Castelli, F., \& Plez, B. 1998, A\&A, 333, 231

Böhm-Vitense, E. 1981, ARA\&A, 19, 295

Burnichon, M. L. 1975, A\&A, 45, 383

Burnichon, M. L., \& Garnier, R. 1976, A\&AS, 24, 89

Cardelli, J. A., Clayton, G. C., \& Mathis, J. S. 1989, ApJ, 345, 245

Carlson, L. R., et al. 2007, ApJ, 665, L109

Cohen, M., Wheaton, W. A., \& Megeath, S. T. 2003, AJ, 126, 1090

Colina, L., Bohlin, R., \& Castelli, F. 1996, HST Instrument Science Report CAL/SCS-008 (Baltimore: STScI)

Collinder, P. 1931, Ann. Obs. Lund, 2, 1

Comerón, F., \& Pasquali, A. 2005, A\&A, 430, 541

Comerón, F., Pasquali, A., Figueras, F., \& Torra, J. 2008, A\&A, 486, 453

Comerón, F., et al. 2002, A\&A, 389, 874

Cutri, R., et al. 2003, 2MASS All-Sky Catalog of Point Sources (Pasadena: IPAC/Caltech)

Droege, T. F., Richmond, M. W., \& Sallman, M. 2006, PASP, 118, 1666

Eaton, J. A. 1995, AJ, 109, 1797

Egan, M. P., \& Price, S. D. 1996, AJ, 112, 2862

Fitzpatrick, E. L. 1999, PASP, 111, 63

Fullerton, A. W. 1990, Ph.D. dissertation, Univ. of Toronto 
Gustafsson, B., Edvardsson, B., Eriksson, K., Jørgensen, U. G., Nordlund, A., \& Plez, B. 2008, A\&A, 486, 951

Hartkopf, W. I., Gies, D. R., Mason, B. D., Bagnuolo, W. G., Jr., \& McAlister, H. A. 1993, BAAS, 25, 872

Hoffleit, D. 1982, The Bright Star Catalogue, 4th ed. (New Haven: Yale Univ. Obs.)

Indebetouw, R., et al. 2005, ApJ, 619, 931

Kharchenko, N. V., Piskunov, A. E., Röser, S., Schilbach, E., \& Scholz, R.-D. 2005, A\&A, 438,1163

Kumar, B., Sagar, R., Sanwal, B. B., \& Bessell, M. S. 2004, MNRAS, 353, 991

Landolt, A. U. 1992, AJ, 104, 340

Lejeune, T., \& Schaerer, D. 2001, A\&A, 366, 538

Lesh, J. R. 1979, in Spectral Classification of the Future (IAU Coll. 47), Ricerche Astronomiche, 9, ed. M. F. McCarthy, A. D. G. Philip, \& G. V. Coyne (Citta del Vaticano: Specola Vaticana), 81

Marigo, P., Girardi, L., Bressan, A., Groenewegen, M. A. T., Silva, L., \& Granato, G. L. 2008, A\&A, 482, 883

Martins, F., Schaerer, D., \& Hillier, D. J. 2005, A\&A, 436, 1049

Mason, B. D., Hartkopf, W. I., Gies, D. R., Henry, T. J., \& Helsel, J. W. 2009, AJ, 137, 3358

Massey, P., DeGioia-Eastwood, K., \& Waterhouse, E. 2001, AJ, 121, 1050

McAlister, H. A., Hartkopf, W. I., Hutter, D. J., Shara, M. M., \& Franz, O. G. 1987, AJ, 92,183

McKibben, W. P., et al. 1998, PASP, 110, 900

Negueruela, I., Marco, A., Israel, G. L., \& Bernabeu, G. 2007, A\&A, 471, 485

Perryman, M. A. C., et al. 1997, A\&A, 323, L49

Reid, I.N., et al. 1991, PASP, 103, 661

Schilbach, E., \& Röser, S. 2008, A\&A, 489, 105 
Schlegel, D. J., Finkbeiner, D. P., \& Davis, M. 1998, ApJ, 500, 525

Schröder, S. E., Kaper, L., Lamers, H. J. G. L. M., \& Brown, A. G. A. 2004, A\&A, 428, 149

Siess, L., Dufour, E., \& Forestini, M. 2000, A\&A, 358, 593

Shatskii, N. I., \& Tokovinin, A. A. 1998, Astronomy Letters, 24, 673

ten Brummelaar, T., Mason, B. D., McAlister, H. A., Roberts, L. C., Jr., Turner, N. H., Hartkopf, W. I., \& Bagnuolo, W. G., Jr. 2000, AJ, 119, 2403

Turner, D. G., \& Forbes, D. 1982, PASP, 94, 789

Turner, N. H., ten Brummelaar, T. A., \& Mason, B. D. 2008, BAAS, 40, 208

Valdes, F., Gupta, R., Rose, J. A., Singh, H. P., \& Bell, D. J. 2004, ApJS, 152, 251

van Belle, G. T., et al. 1999, AJ, 117, 521

van Leeuwen, F. 2007, A\&A, 474, 653

Walborn, N. R. 1972, AJ, 77, 312

Walborn, N.R., \& Fitzpatrick, E.L. 1990, PASP, 102, 379

Wegner, W. 1994, MNRAS, 270, 229

Zacharias, N., et al. 2010, AJ, 139, 2184 


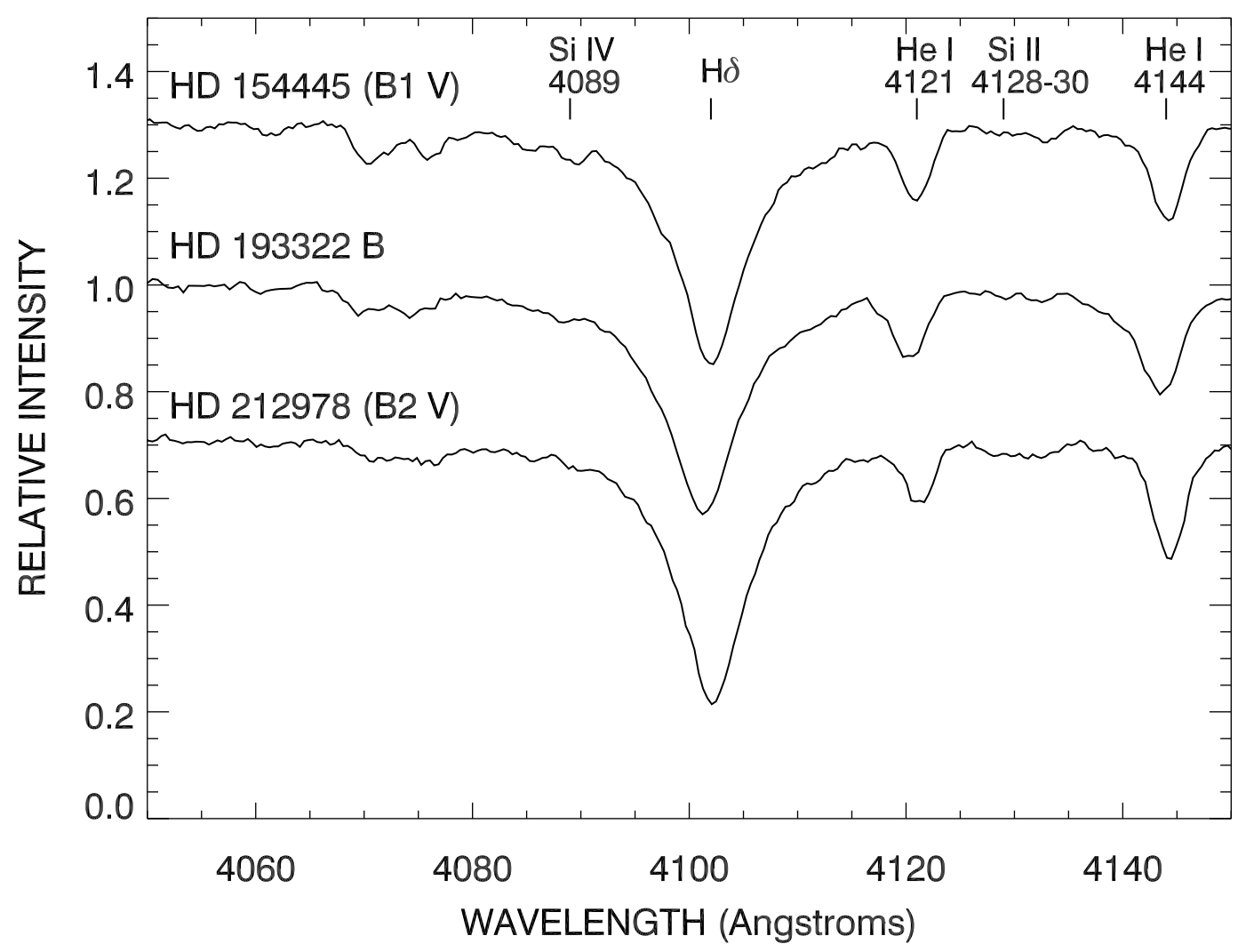

Fig. 1.- Our estimate for the spectral type of HD 193322 B is B1.5 V based on comparisons to stars in Valdes et al. (2004). Walborn \& Fitzpatrick (1990) advocate using the Si lines to classify early B stars, and those lines were given the strongest weight. The spectra were normalized and then offset to improve clarity. 


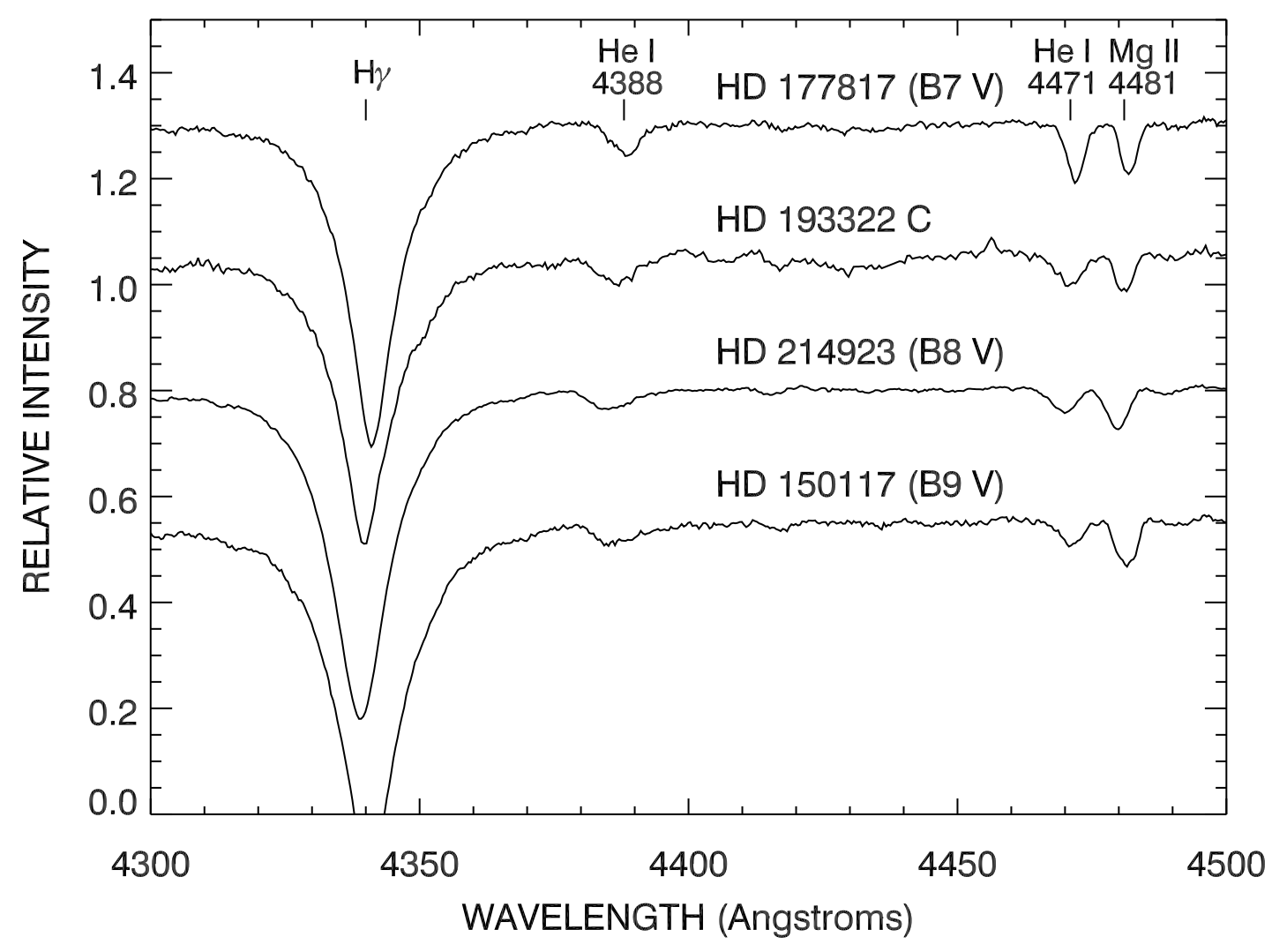

Fig. 2.- We estimate the spectral type of HD $193322 \mathrm{C}$ as B8 V or possibly B9 V. The spectra were normalized and then offset to improve clarity. 


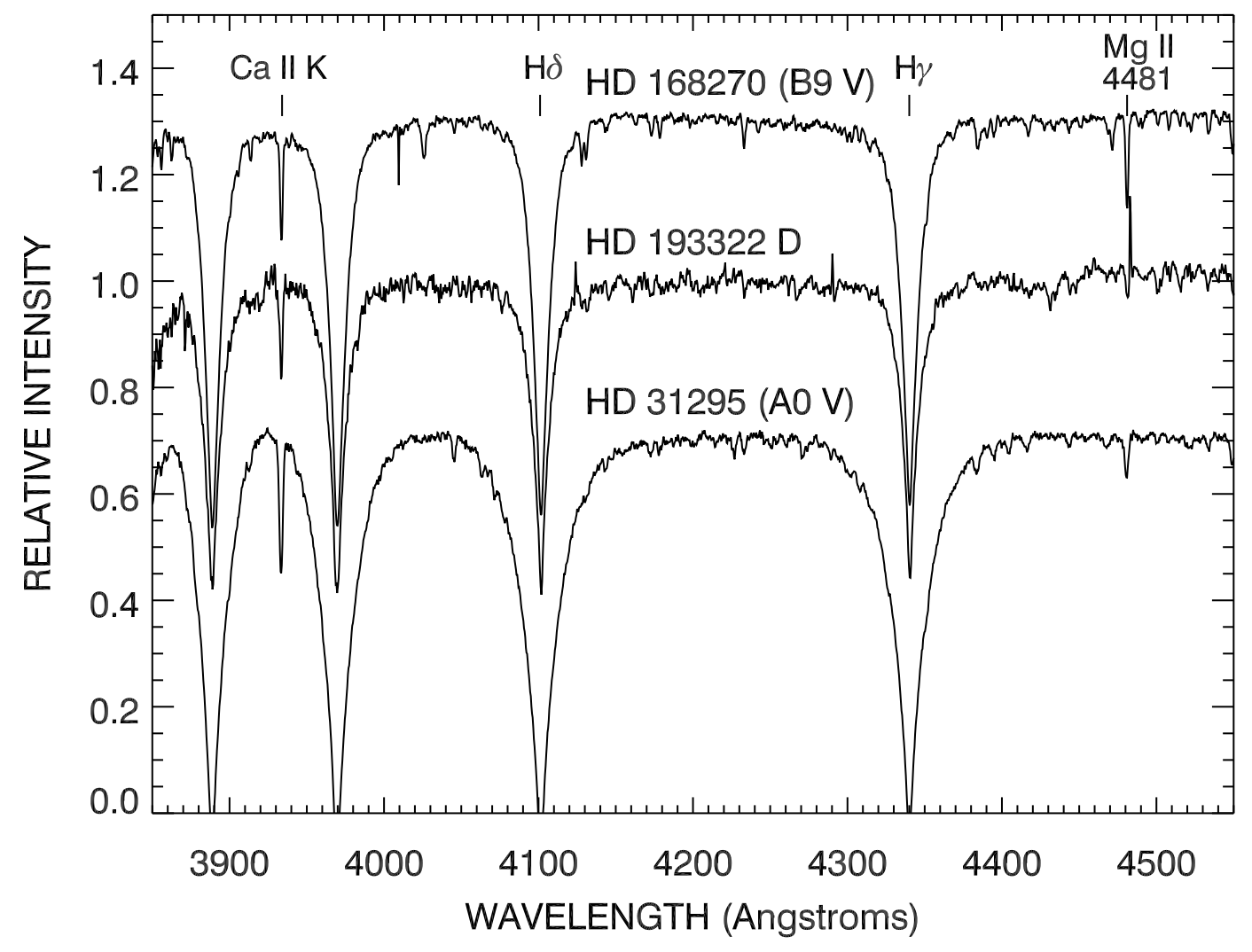

Fig. 3.- We estimate the spectral type of HD 193322 D as B9 V due to the strength of the Ca II K lines. The H Balmer, He, and metal lines are weak. The spectra were normalized and then offset to improve clarity. 


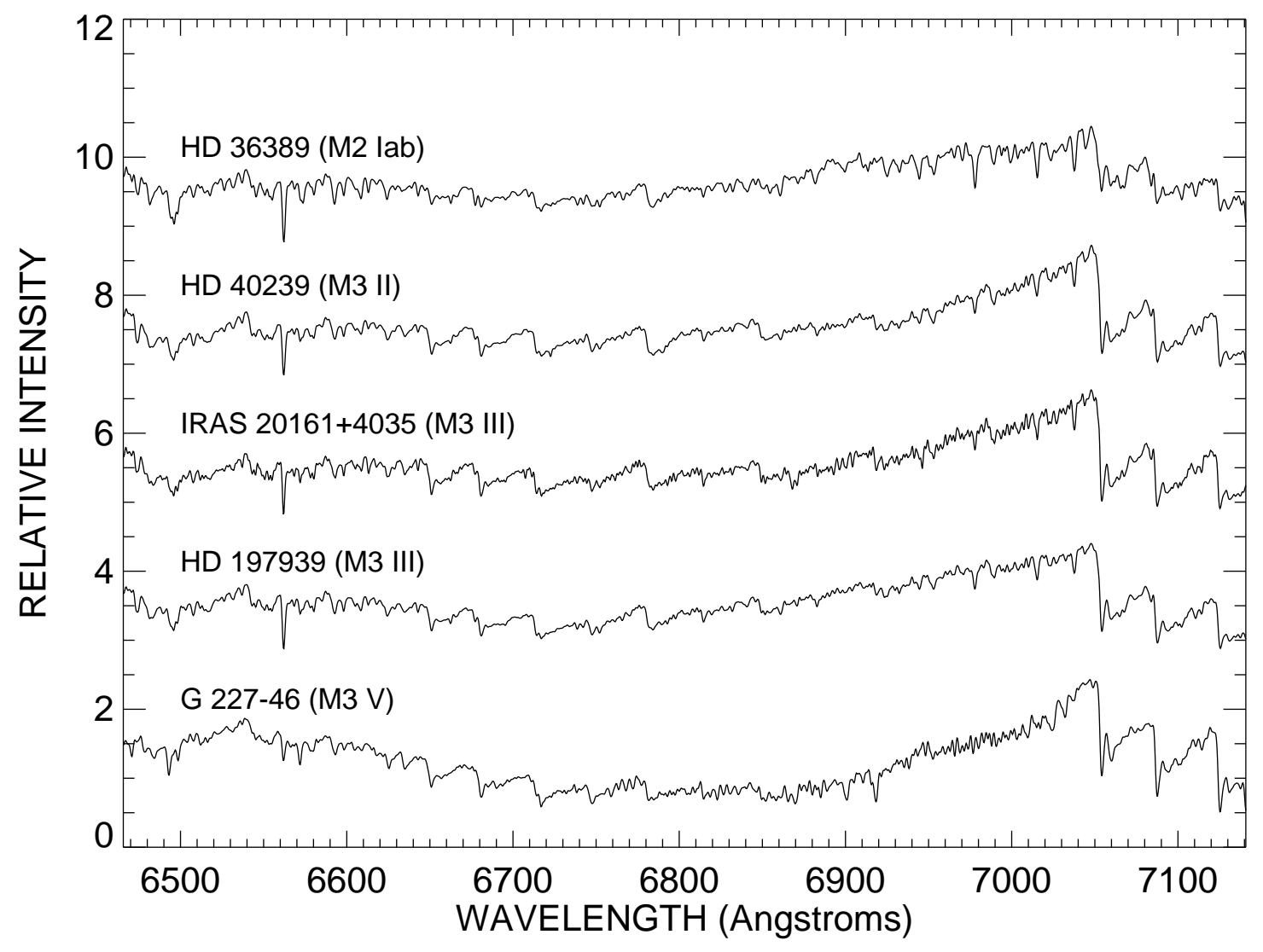

Fig. 4. - A comparison of the red spectrum of IRAS 20161+4035 with similar spectra of cool stars from the atlas of Valdes et al. (2004). Each spectrum has been offset in relative intensity for clarity of presentation. 


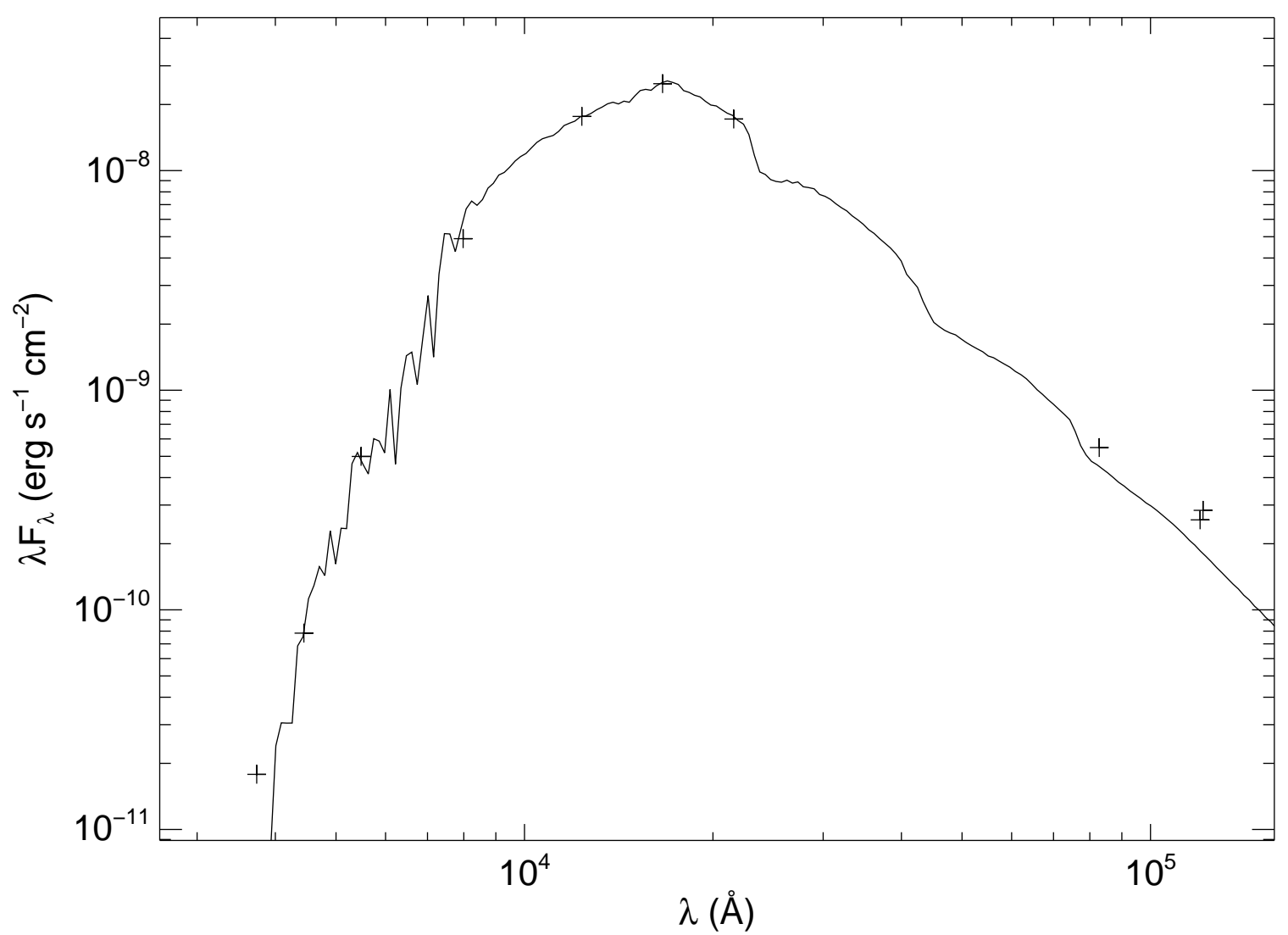

Fig. 5.- The spectral energy distribution of IRAS20161+4035 (plus signs) compared with a reddened model SED (solid line). 


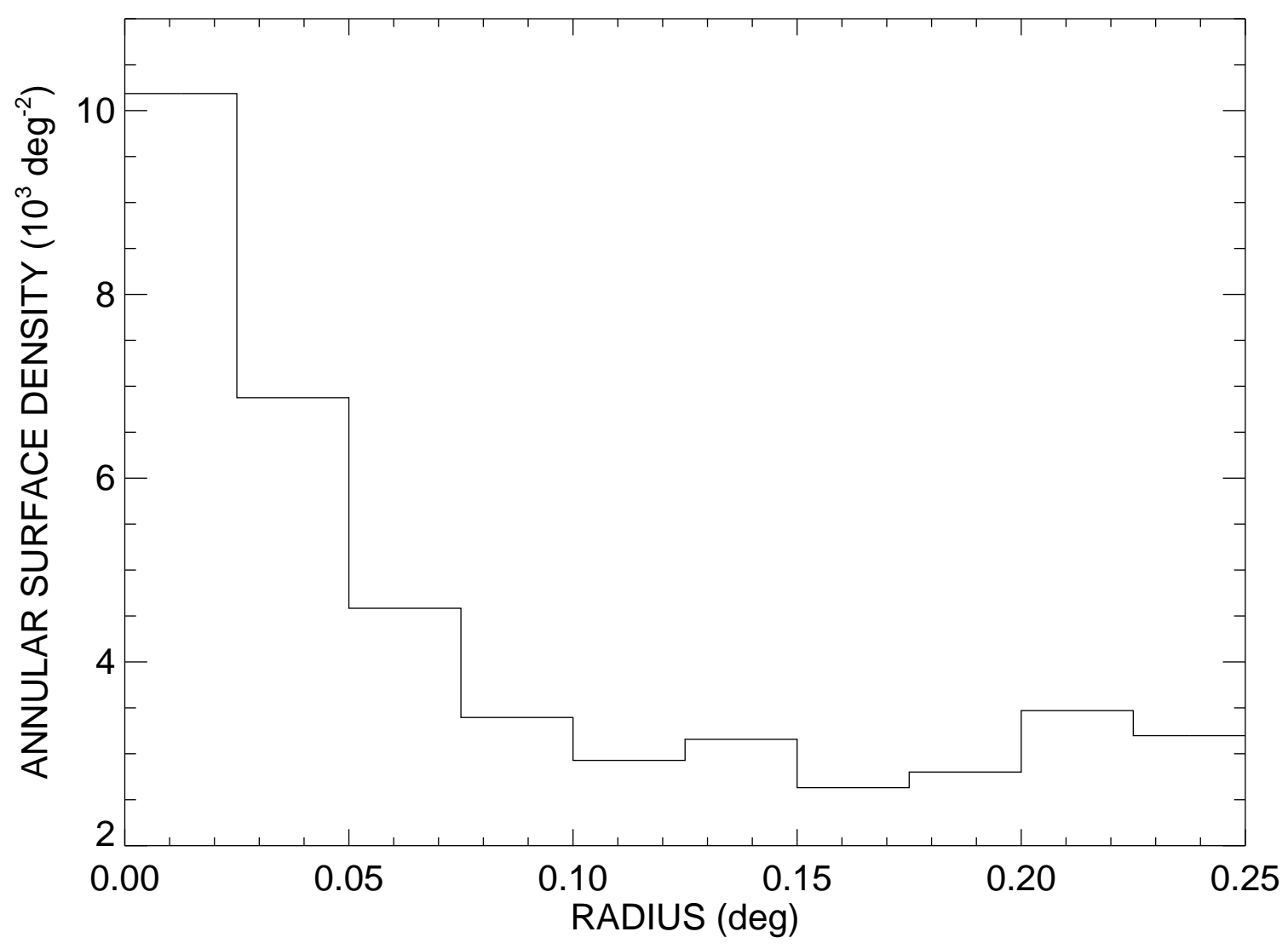

Fig. 6. - The distribution of the stellar number density per unit area in rings of increasing radius centered on HD 193322, the nominal center of $\mathrm{Cr} 419$. 


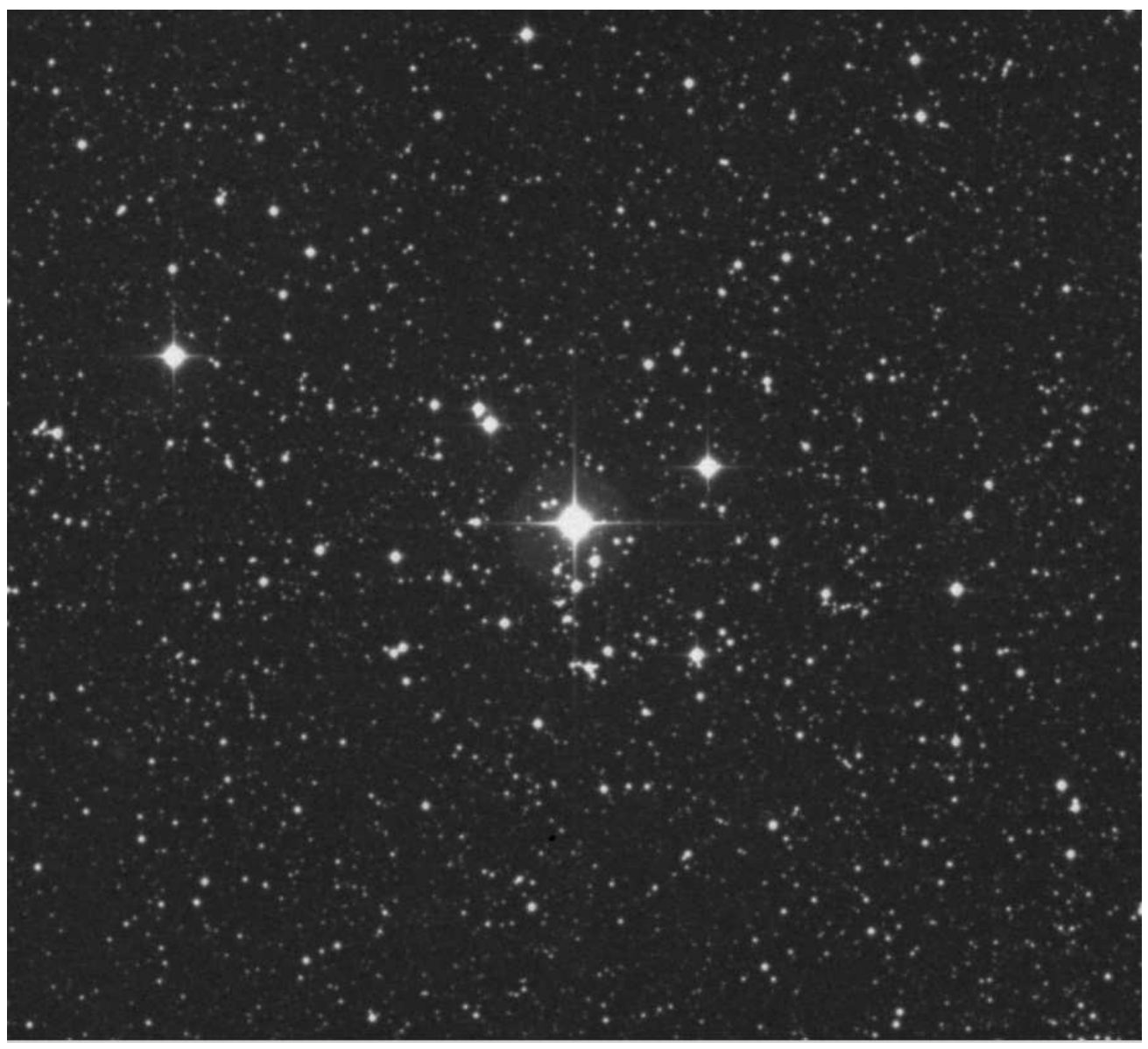

Fig. 7. - The POSS2-IR image centered on HD 193322 with a field of view of a $0.25^{\circ} \times 0.25^{\circ}$ showing the low stellar density of the cluster. North is up and east is to the left. 


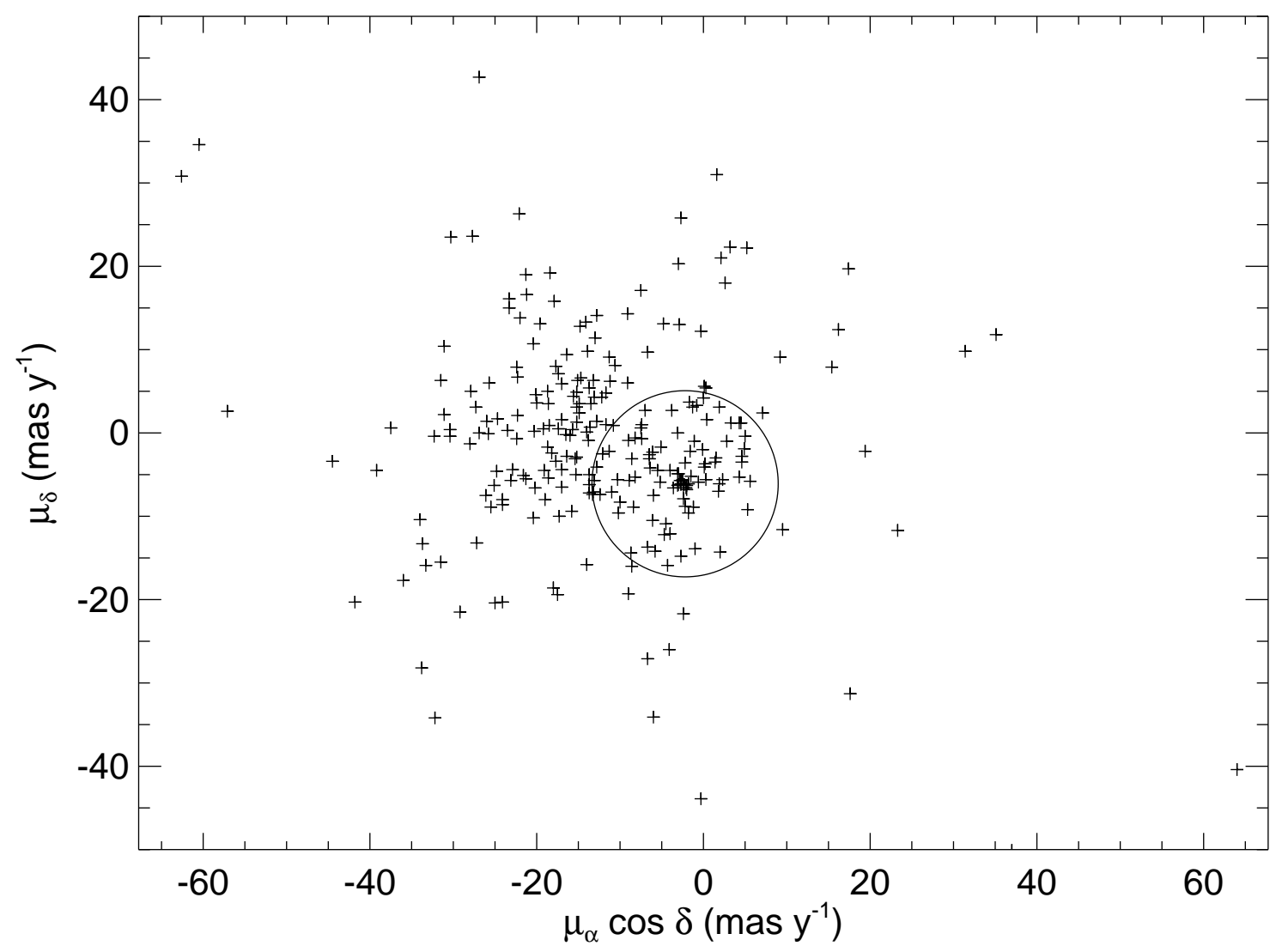

Fig. 8. - The distribution of proper motions in right ascension $\left(\mu_{\alpha} \cos \delta\right)$ and declination $\left(\mu_{\delta}\right)$ for stars within $0: 16$ of the cluster center. The position of the circle marks the cluster average proper motion and its circumference represents the boundary for possible cluster membership. 


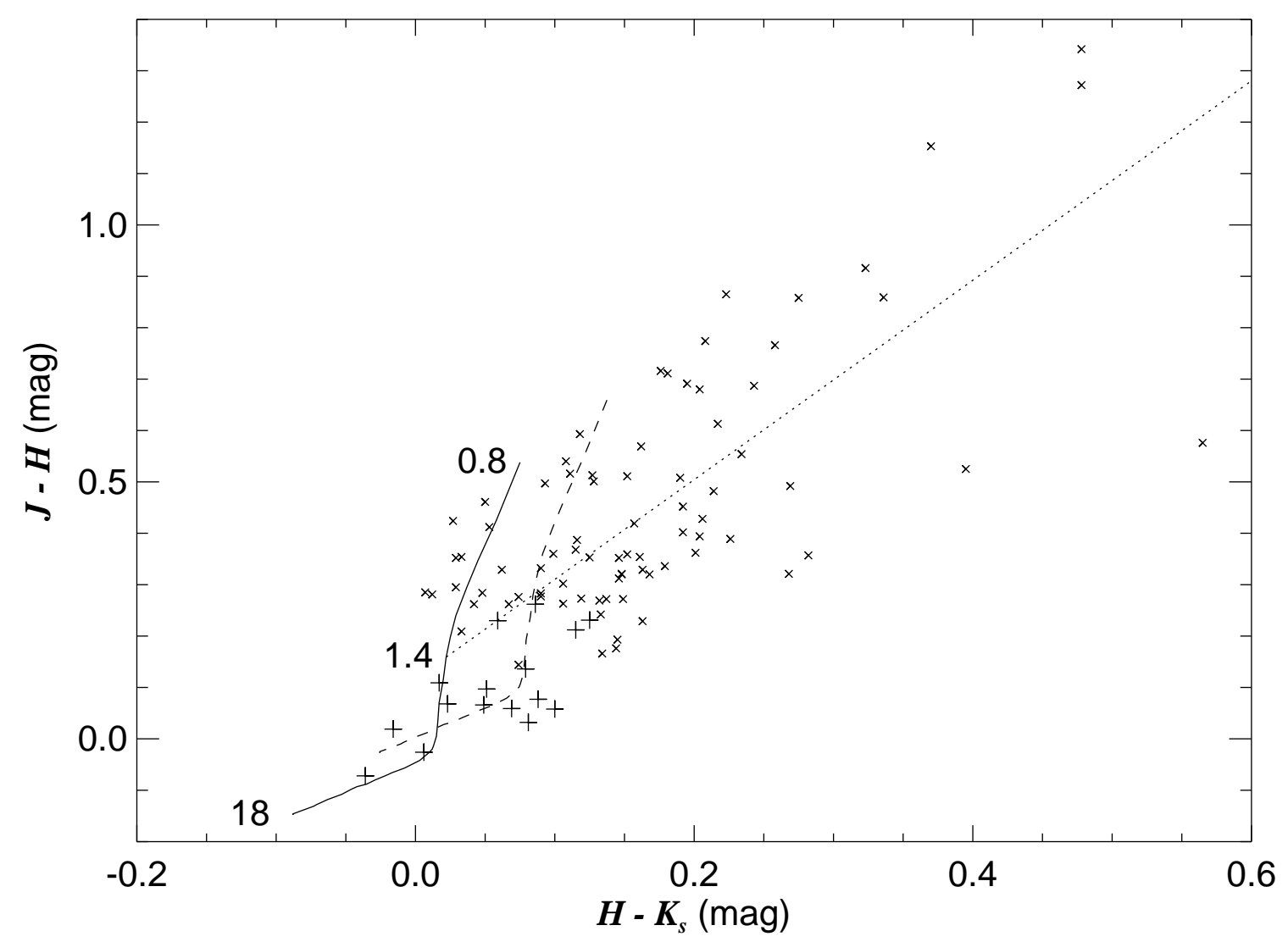

Fig. 9. - The distribution of near-IR colors for those stars meeting the positional and proper motion criteria for cluster membership. Plus signs mark those that are probable, main sequence members while $\times$ signs are used for the rest of the sample. The solid and dashed lines represent the values for the cluster isochrone for no reddening and $E(B-V)=0.37$, respectively. The numbers beside the unreddened isochrone indicate the stellar masses at the terminal points and at the origin of the reddening trajectory (dotted line). Stars below the dotted line are candidate massive stars $\left(M>1.4 M_{\odot}\right)$. 


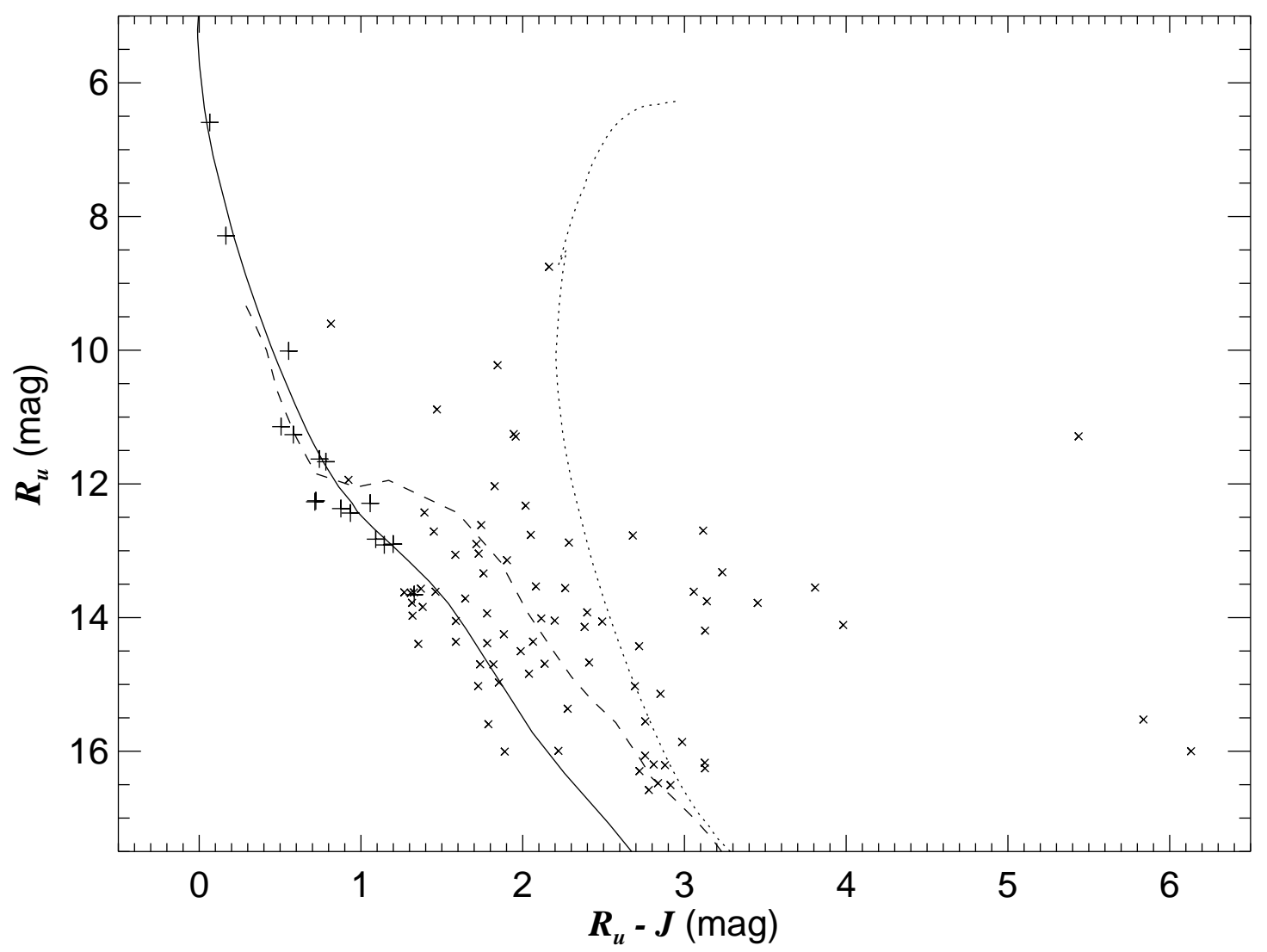

Fig. 10.- A color-magnitude diagram based upon the UCAC3 $R_{u}$ and 2MASS $J$ magnitudes. Plus signs mark probable, main sequence members, while $\times$ symbols are used for the remainder of the sample that meets the positional and proper motion criteria for cluster membership. The solid line shows the best fit isochrone. The dashed line indicates the pre-main sequence isochrone for an age of $7 \mathrm{Myr}$. The dotted line is the isochrone for distant OB stars that may be related to the nearby cluster Berkeley 87 . 


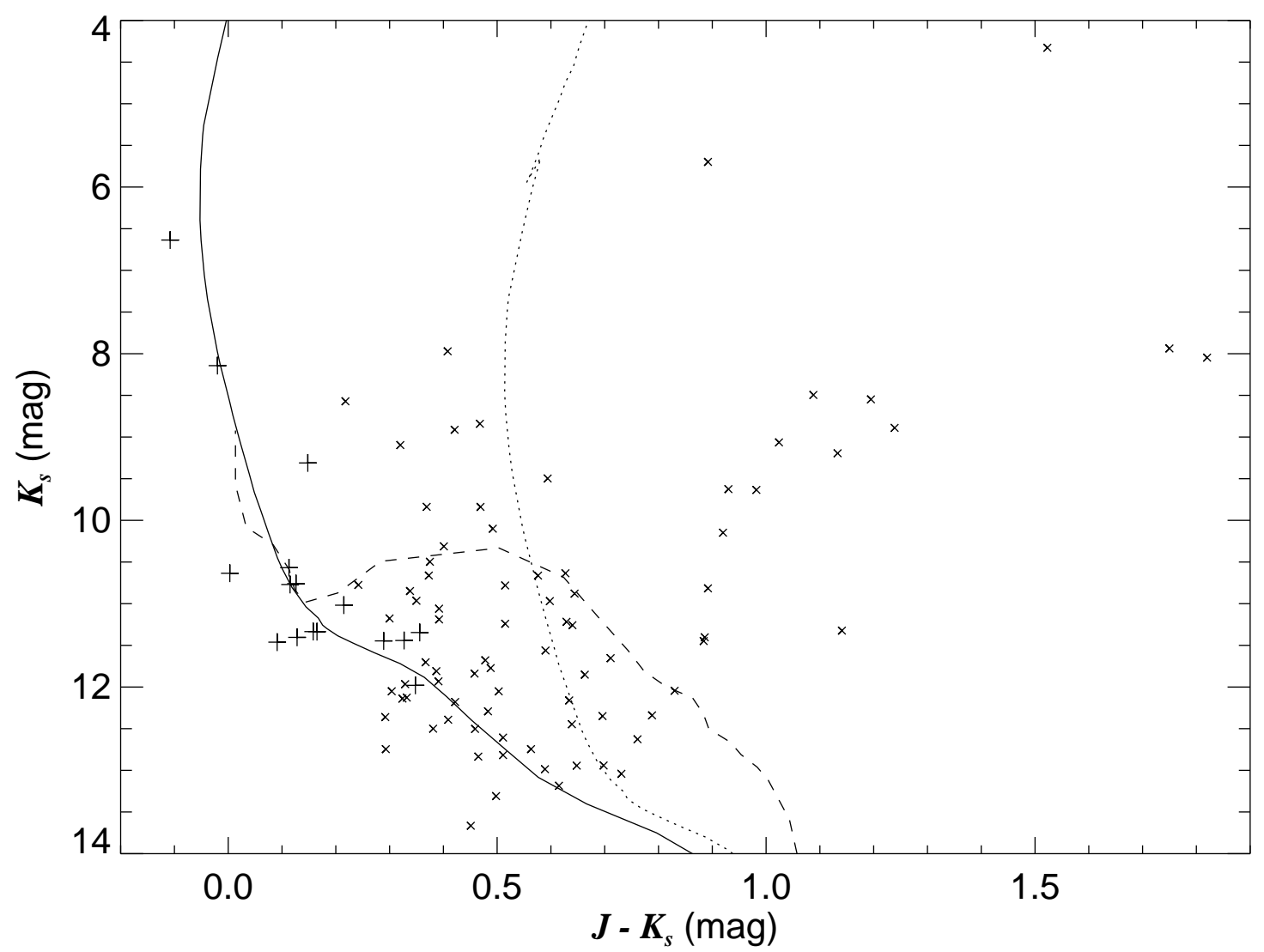

Fig. 11.- A color-magnitude diagram for 2MASS $J$ and $K_{s}$ magnitudes (in the same format as Fig. (10). The bright, red object (top, right) is IRAS 20161+4035. 


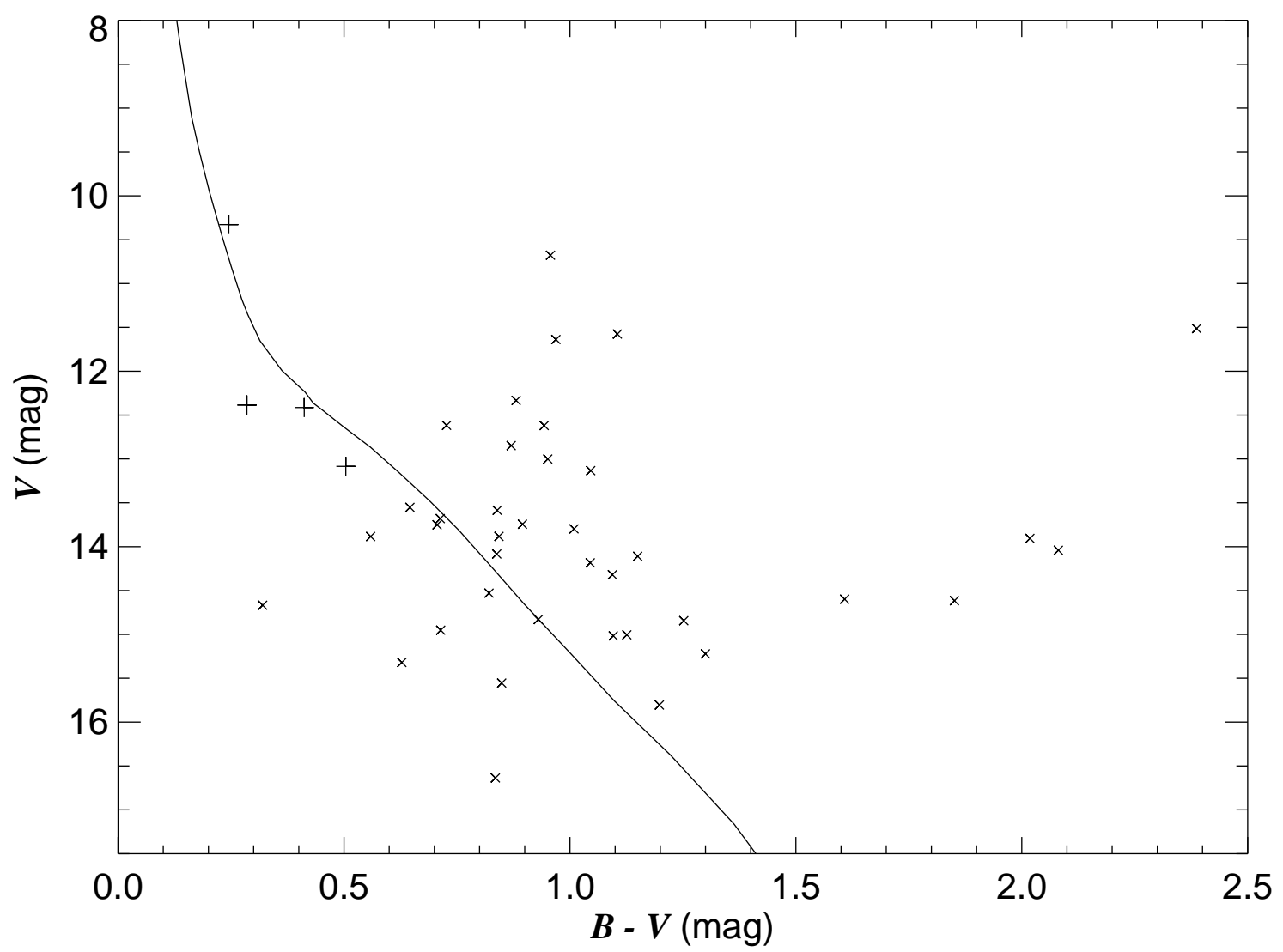

Fig. 12. - A color-magnitude diagram from optical photometry in the same format as Fig. 10. The solid line shows the isochrone for our derived $E(B-V)$ and DM. 


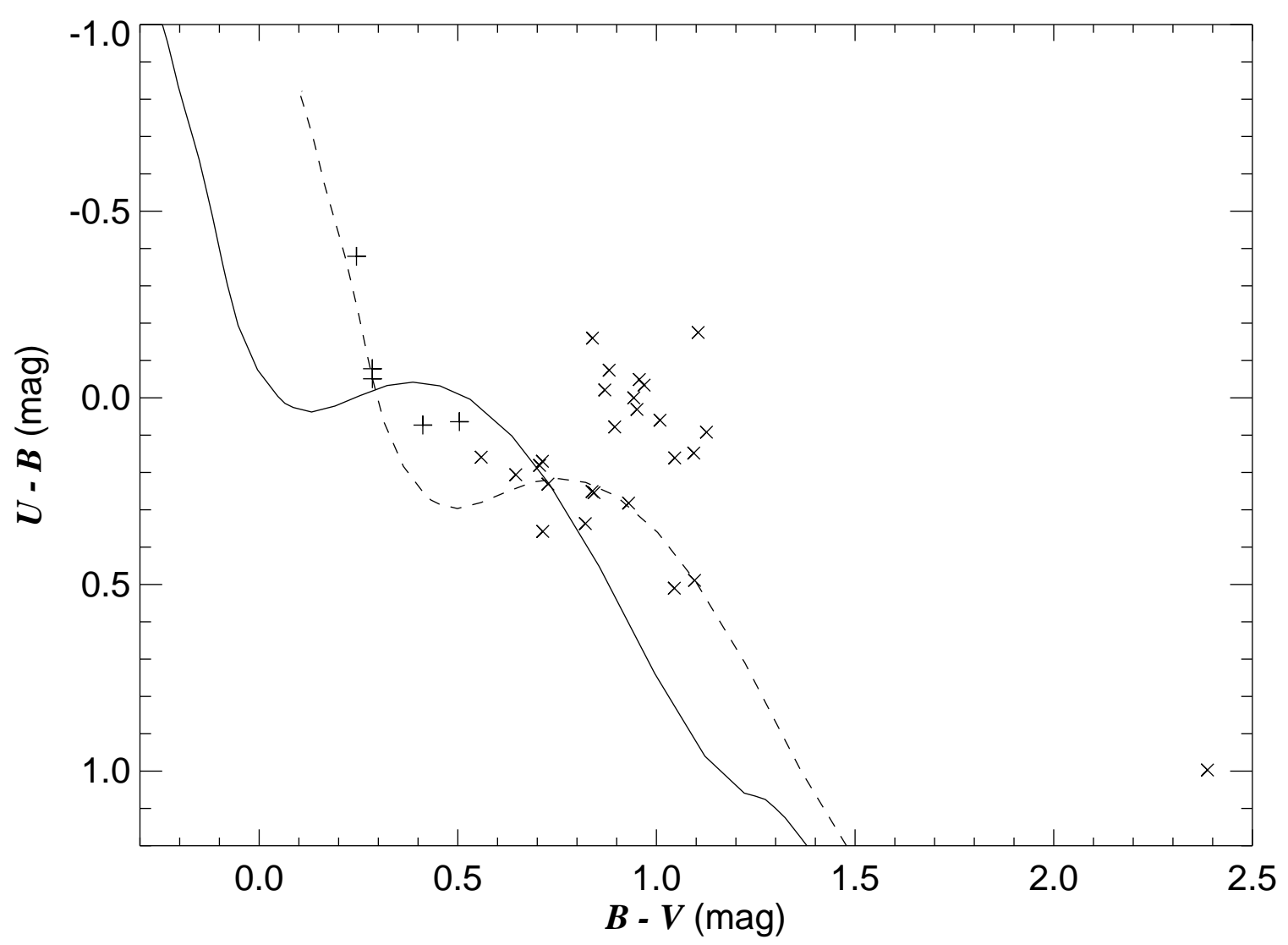

Fig. 13.- A color-color diagram from optical photometry in a format similar to Fig. 9. The solid and dashed lines show the model unreddened and reddened colors, respectively. 
Table 1. Reddening Estimates for $R=3.1$

\begin{tabular}{ccccc}
\hline \hline $\begin{array}{c}\text { Star } \\
\text { Name }\end{array}$ & $\begin{array}{c}\text { Spectral } \\
\text { Classification }\end{array}$ & $\begin{array}{c}E(B-V) \\
(\mathrm{mag})\end{array}$ & $\begin{array}{c}\theta_{L D} \\
(\mu \mathrm{as})\end{array}$ & $\begin{array}{c}d \\
(\mathrm{kpc})\end{array}$ \\
\hline HD 193322Aa1 $\ldots$ & O9 V:((n)) & $0.31 \pm 0.02$ & $85 \pm 2$ & 0.83 \\
HD 193322B $\ldots .$. & B1.5 V & $0.28 \pm 0.03$ & $53 \pm 2$ & 0.73 \\
HD 193322C $\ldots .$. & B8 V & $0.29 \pm 0.02$ & $23 \pm 1$ & 0.79 \\
HD 193322D $\ldots . .$. & B9 V & $0.29 \pm 0.03$ & $25 \pm 1$ & 0.70 \\
IRAS 20161+4035 & M3 III & $0.74 \pm 0.04$ & $893 \pm 46$ & 0.78 \\
\hline
\end{tabular}

Table 2. Adopted Flux Ratios for the Components of HD 193322

\begin{tabular}{|c|c|c|c|c|c|c|c|c|}
\hline $\begin{array}{c}\text { Star } \\
\text { Name }\end{array}$ & $\begin{array}{l}T_{\text {eff }} \\
(\mathrm{kK})\end{array}$ & $\begin{array}{c}F_{x} / F_{A a 1} \\
B\end{array}$ & $\begin{array}{c}F_{x} / F_{A a 1} \\
V\end{array}$ & $\begin{array}{c}F_{x} / F_{A a 1} \\
R_{j}\end{array}$ & $\begin{array}{c}F_{x} / F_{A a 1} \\
I_{j}\end{array}$ & $\begin{array}{c}F_{x} / F_{A a 1} \\
J\end{array}$ & $\begin{array}{c}F_{x} / F_{A a 1} \\
H\end{array}$ & $\begin{array}{c}F_{x} / F_{A a 1} \\
K_{s}\end{array}$ \\
\hline HD 193322Aa1 & 33.2 & 1.00 & 1.00 & 1.00 & 1.00 & 1.00 & 1.00 & 1.00 \\
\hline HD 193322Aa2 & 23.0 & 0.29 & 0.30 & 0.31 & 0.33 & 0.33 & 0.35 & 0.35 \\
\hline HD 193322Ab. & 25.0 & 0.41 & 0.43 & 0.44 & 0.46 & 0.47 & 0.48 & 0.48 \\
\hline HD 193322B.. & 23.0 & 0.21 & 0.21 & 0.21 & 0.21 & 0.23 & 0.24 & 0.25 \\
\hline
\end{tabular}

Table 3. Main Sequence Cluster Members

\begin{tabular}{cccccrrr}
\hline \hline $\begin{array}{c}\text { UCAC3 } \\
\text { Number }\end{array}$ & $\begin{array}{c}\text { Other } \\
\text { Name }\end{array}$ & $\begin{array}{c}R_{u} \\
(\mathrm{mag})\end{array}$ & $\begin{array}{c}R_{u}-J \\
(\mathrm{mag})\end{array}$ & $\begin{array}{c}J-H \\
(\mathrm{mag})\end{array}$ & $\begin{array}{r}H-K_{s} \\
(\mathrm{mag})\end{array}$ & $\begin{array}{c}K_{s} \\
(\mathrm{mag})\end{array}$ & $\begin{array}{c}\text { Mass } \\
\left(M_{\odot}\right)\end{array}$ \\
\hline $262-202014$ & $\ldots$ & 12.29 & 1.06 & 0.14 & 0.08 & 11.02 & 2.2 \\
$262-202033$ & $\ldots$ & 12.90 & 1.20 & 0.23 & 0.12 & 11.35 & 1.7 \\
$262-202070$ & HD 228810 & 10.01 & 0.55 & 0.10 & 0.05 & 9.31 & 5.0 \\
$262-202090$ & $\ldots$ & 12.44 & 0.94 & 0.08 & 0.09 & 11.34 & 1.9 \\
$262-202099$ & $\ldots$ & 13.66 & 1.33 & 0.26 & 0.09 & 11.98 & 1.4 \\
$262-202103$ & $\ldots$ & 12.91 & 1.14 & 0.21 & 0.12 & 11.44 & 1.7 \\
$262-202123$ & HD 193322C & 11.14 & 0.51 & 0.02 & -0.02 & 10.63 & 2.8 \\
$262-202132$ & HD $193322 \mathrm{D}$ & 11.26 & 0.58 & 0.03 & 0.08 & 10.57 & 2.8 \\
$262-202133$ & HD 193322Aa1 & 6.59 & 0.07 & -0.07 & -0.04 & 6.64 & 14.9 \\
$262-202133$ & HD 193322B & 8.29 & 0.17 & -0.03 & 0.01 & 8.14 & 9.0 \\
$262-202155$ & $\ldots$ & 12.27 & 0.71 & 0.07 & 0.02 & 11.46 & 1.8 \\
$262-202269$ & $\ldots$ & 12.25 & 0.72 & 0.06 & 0.07 & 11.40 & 1.8 \\
$262-202314$ & $\ldots$ & 12.83 & 1.09 & 0.23 & 0.06 & 11.45 & 1.7 \\
$262-202350$ & $\ldots$ & 12.37 & 0.88 & 0.06 & 0.10 & 11.34 & 1.9 \\
$262-202365$ & $\ldots$ & 11.63 & 0.74 & 0.07 & 0.05 & 10.77 & 2.5 \\
$262-202402$ & $\ldots$ & 11.67 & 0.78 & 0.11 & 0.02 & 10.76 & 2.5 \\
\hline
\end{tabular}


Table 4. UBV Photometry

\begin{tabular}{|c|c|c|c|c|}
\hline $\begin{array}{l}\text { UCAC3 } \\
\text { Number }\end{array}$ & $\begin{array}{c}U \\
(\mathrm{mag})\end{array}$ & $\begin{array}{c}B \\
(\mathrm{mag})\end{array}$ & $\begin{array}{c}V \\
(\mathrm{mag})\end{array}$ & Member \\
\hline 262-201944 & $12.51 \pm 0.04$ & $12.68 \pm 0.02$ & $11.58 \pm 0.01$ & $\ldots$ \\
\hline 262-201945 & $14.26 \pm 0.04$ & $14.42 \pm 0.02$ & $13.59 \pm 0.02$ & $\ldots$ \\
\hline $262-201948$ & $\ldots$ & $15.92 \pm 0.03$ & $13.91 \pm 0.02$ & $\ldots$ \\
\hline 262-201953 & $\ldots$ & $16.52 \pm 0.04$ & $15.21 \pm 0.01$ & $\ldots$ \\
\hline $262-201963$ & $16.25 \pm 0.05$ & $16.09 \pm 0.03$ & $14.91 \pm 0.02$ & $\ldots$ \\
\hline $262-201973$ & $16.22 \pm 0.06$ & $16.13 \pm 0.04$ & $15.01 \pm 0.02$ & $\ldots$ \\
\hline $262-201978$ & $16.60 \pm 0.06$ & $16.11 \pm 0.04$ & $15.02 \pm 0.02$ & $\ldots$ \\
\hline $262-201981$ & $15.30 \pm 0.04$ & $15.27 \pm 0.02$ & $14.43 \pm 0.01$ & $\ldots$ \\
\hline 262-201993 & $16.04 \pm 0.05$ & $15.76 \pm 0.02$ & $14.83 \pm 0.02$ & $\ldots$ \\
\hline 262-201997 & $\ldots$ & $16.66 \pm 0.03$ & $15.53 \pm 0.03$ & $\ldots$ \\
\hline 262-202009 & $\ldots$ & $17.15 \pm 0.05$ & $15.86 \pm 0.01$ & $\cdots$ \\
\hline $262-202014$ & $12.90 \pm 0.04$ & $12.83 \pm 0.01$ & $12.41 \pm 0.03$ & Yes \\
\hline 262-202017 & $15.74 \pm 0.04$ & $15.23 \pm 0.02$ & $14.18 \pm 0.01$ & $\ldots$ \\
\hline $262-202018$ & $\ldots$ & $14.99 \pm 0.04$ & $14.67 \pm 0.03$ & $\ldots$ \\
\hline $262-202025$ & $\ldots$ & $16.47 \pm 0.03$ & $14.62 \pm 0.01$ & $\ldots$ \\
\hline $262-202026$ & $\ldots$ & $16.31 \pm 0.03$ & $15.22 \pm 0.01$ & $\ldots$ \\
\hline $262-202027$ & $\ldots$ & $16.49 \pm 0.03$ & $15.61 \pm 0.01$ & $\cdots$ \\
\hline 262-202032 & $14.72 \pm 0.04$ & $14.64 \pm 0.01$ & $13.74 \pm 0.01$ & $\ldots$ \\
\hline $262-202033$ & $13.65 \pm 0.04$ & $13.59 \pm 0.01$ & $13.08 \pm 0.02$ & Yes \\
\hline $262-202034$ & $\ldots$ & $16.12 \pm 0.02$ & $14.04 \pm 0.02$ & $\ldots$ \\
\hline 262-202039 & $15.17 \pm 0.04$ & $14.92 \pm 0.01$ & $14.08 \pm 0.01$ & $\ldots$ \\
\hline 262-202042 & $\ldots$ & $15.95 \pm 0.02$ & $15.32 \pm 0.02$ & $\ldots$ \\
\hline $262-202045$ & $14.40 \pm 0.04$ & $14.20 \pm 0.03$ & $13.55 \pm 0.03$ & $\ldots$ \\
\hline 262-202046 & $\ldots$ & $16.88 \pm 0.04$ & $15.73 \pm 0.01$ & $\ldots$ \\
\hline $262-202047$ & $\ldots$ & $17.26 \pm 0.05$ & $15.96 \pm 0.01$ & $\ldots$ \\
\hline 262-202050 & $\ldots$ & $16.52 \pm 0.03$ & $15.22 \pm 0.01$ & $\cdots$ \\
\hline $262-202052$ & $15.33 \pm 0.04$ & $14.99 \pm 0.02$ & $14.20 \pm 0.03$ & $\ldots$ \\
\hline $262-202054$ & $16.21 \pm 0.05$ & $15.74 \pm 0.02$ & $14.76 \pm 0.02$ & $\ldots$ \\
\hline 262-202059 & $14.90 \pm 0.04$ & $13.90 \pm 0.01$ & $11.51 \pm 0.04$ & $\ldots$ \\
\hline $262-202061$ & $\ldots$ & $17.47 \pm 0.05$ & $16.64 \pm 0.01$ & $\ldots$ \\
\hline 262-202066 & $14.98 \pm 0.04$ & $14.73 \pm 0.02$ & $13.88 \pm 0.03$ & $\ldots$ \\
\hline $262-202067$ & $14.64 \pm 0.04$ & $14.46 \pm 0.01$ & $13.75 \pm 0.02$ & $\ldots$ \\
\hline 262-202070 & $10.19 \pm 0.04$ & $10.57 \pm 0.01$ & $10.33 \pm 0.01$ & Yes \\
\hline $262-202088$ & $13.09 \pm 0.04$ & $13.03 \pm 0.01$ & $12.48 \pm 0.02$ & $\ldots$ \\
\hline 262-202089 & $16.76 \pm 0.07$ & $16.24 \pm 0.03$ & $15.13 \pm 0.02$ & $\ldots$ \\
\hline $262-202097$ & $\ldots$ & $16.21 \pm 0.03$ & $14.60 \pm 0.01$ & $\ldots$ \\
\hline $262-202098$ & $15.69 \pm 0.05$ & $15.35 \pm 0.02$ & $14.53 \pm 0.01$ & $\ldots$ \\
\hline $262-202100$ & $\ldots$ & $17.24 \pm 0.05$ & $15.92 \pm 0.02$ & $\cdots$ \\
\hline 262-202107 & $16.02 \pm 0.05$ & $15.67 \pm 0.02$ & $14.95 \pm 0.02$ & $\ldots$ \\
\hline 262-202109 & $\ldots$ & $16.33 \pm 0.03$ & $15.31 \pm 0.02$ & $\ldots$ \\
\hline $262-202117$ & $13.98 \pm 0.04$ & $13.95 \pm 0.01$ & $13.00 \pm 0.02$ & $\ldots$ \\
\hline 262-202118 & $\ldots$ & $16.08 \pm 0.05$ & $15.53 \pm 0.01$ & $\cdots$ \\
\hline $262-202120$ & $\ldots$ & $15.26 \pm 0.02$ & $14.11 \pm 0.02$ & $\ldots$ \\
\hline 262-202128 & $13.56 \pm 0.04$ & $13.56 \pm 0.01$ & $12.62 \pm 0.01$ & $\ldots$ \\
\hline 262-202135 & $16.41 \pm 0.05$ & $16.15 \pm 0.02$ & $15.12 \pm 0.01$ & $\ldots$ \\
\hline
\end{tabular}


Table 4-Continued

\begin{tabular}{ccccc}
\hline \hline UCAC3 & $\begin{array}{c}\text { U } \\
\text { Number }\end{array}$ & $\begin{array}{c}B \\
(\mathrm{mag})\end{array}$ & $\begin{array}{c}V \\
(\mathrm{mag})\end{array}$ & Member \\
\hline $262-202141$ & $16.66 \pm 0.09$ & $15.72 \pm 0.03$ & $14.48 \pm 0.01$ & $\ldots$ \\
$262-202146$ & $\ldots$ & $16.35 \pm 0.03$ & $15.13 \pm 0.04$ & $\ldots$ \\
$262-202155$ & $12.62 \pm 0.04$ & $12.67 \pm 0.01$ & $12.39 \pm 0.03$ & Yes \\
$262-202156$ & $\ldots$ & $16.79 \pm 0.03$ & $15.72 \pm 0.02$ & $\ldots$ \\
$262-202159$ & $16.45 \pm 0.05$ & $16.09 \pm 0.02$ & $15.08 \pm 0.01$ & $\ldots$ \\
$262-202161$ & $\ldots$ & $17.00 \pm 0.03$ & $16.07 \pm 0.02$ & $\ldots$ \\
$262-202166$ & $14.60 \pm 0.04$ & $14.44 \pm 0.02$ & $13.88 \pm 0.02$ & $\ldots$ \\
$262-202170$ & $11.59 \pm 0.04$ & $11.63 \pm 0.01$ & $10.68 \pm 0.03$ & $\ldots$ \\
$262-202175$ & $\ldots$ & $17.59 \pm 0.08$ & $16.33 \pm 0.05$ & $\ldots$ \\
$262-202176$ & $12.57 \pm 0.04$ & $12.61 \pm 0.01$ & $11.64 \pm 0.01$ & $\ldots$ \\
$262-202177$ & $15.22 \pm 0.04$ & $14.70 \pm 0.02$ & $13.65 \pm 0.04$ & $\ldots$ \\
$262-202178$ & $\ldots$ & $16.73 \pm 0.04$ & $15.81 \pm 0.01$ & $\ldots$ \\
$262-202185$ & $\ldots$ & $17.45 \pm 0.06$ & $16.09 \pm 0.01$ & $\ldots$ \\
$262-202187$ & $\ldots$ & $17.41 \pm 0.06$ & $16.30 \pm 0.01$ & $\ldots$ \\
$262-202190$ & $14.87 \pm 0.04$ & $14.81 \pm 0.01$ & $13.80 \pm 0.01$ & $\ldots$ \\
$262-202194$ & $15.97 \pm 0.05$ & $15.68 \pm 0.02$ & $14.71 \pm 0.03$ & $\ldots$ \\
$262-202197$ & $\ldots$ & $16.89 \pm 0.04$ & $15.79 \pm 0.01$ & $\ldots$ \\
$262-202199$ & $14.34 \pm 0.04$ & $14.18 \pm 0.01$ & $13.13 \pm 0.01$ & $\ldots$ \\
$262-202207$ & $14.56 \pm 0.04$ & $14.39 \pm 0.01$ & $13.68 \pm 0.01$ & $\ldots$ \\
$262-202212$ & $15.56 \pm 0.04$ & $15.41 \pm 0.02$ & $14.32 \pm 0.02$ & $\ldots$ \\
$262-202218$ & $13.14 \pm 0.04$ & $13.21 \pm 0.01$ & $12.33 \pm 0.01$ & $\ldots$ \\
$262-202225$ & $\ldots$ & $16.96 \pm 0.04$ & $15.83 \pm 0.02$ & $\ldots$ \\
$262-202224$ & $\ldots$ & $16.75 \pm 0.03$ & $15.66 \pm 0.01$ & $\ldots$ \\
$262-202227$ & $16.31 \pm 0.05$ & $15.84 \pm 0.02$ & $14.89 \pm 0.06$ & $\ldots$ \\
$262-202236$ & $16.47 \pm 0.05$ & $16.18 \pm 0.03$ & $15.41 \pm 0.01$ & $\ldots$ \\
$262-202239$ & $\ldots$ & $17.00 \pm 0.04$ & $15.81 \pm 0.04$ & $\ldots$ \\
$262-202243$ & $16.16 \pm 0.05$ & $15.65 \pm 0.02$ & $14.80 \pm 0.01$ & $\ldots$ \\
$262-202247$ & $13.70 \pm 0.04$ & $13.72 \pm 0.01$ & $12.85 \pm 0.03$ & $\ldots$ \\
$262-202248$ & $13.57 \pm 0.04$ & $13.34 \pm 0.02$ & $12.62 \pm 0.07$ & $\ldots$ \\
$262-202255$ & $\ldots$ & $16.40 \pm 0.03$ & $15.56 \pm 0.01$ & $\ldots$ \\
$262-202256$ & $\ldots$ & $17.86 \pm 0.10$ & $16.15 \pm 0.03$ & $\ldots$ \\
$262-202260$ & $\ldots$ & $16.10 \pm 0.03$ & $14.84 \pm 0.01$ & $\ldots$ \\
$262-202265$ & $14.56 \pm 0.04$ & $14.08 \pm 0.02$ & $13.27 \pm 0.01$ & $\ldots$ \\
$262-202269$ & $12.59 \pm 0.04$ & $12.67 \pm 0.02$ & $12.38 \pm 0.02$ & Yes \\
\hline & & & &
\end{tabular}

